\title{
Manuscript title: Evaluating the role of alcohol consumption in breast and ovarian cancer susceptibility using population-based cohort studies and two-sample Mendelian randomization analyses
}

\section{Authors:}

Jue-Sheng Ong ${ }^{1,2}$, Eske M. Derks ${ }^{1}$, Mikael Eriksson ${ }^{3}$, Jiyuan An ${ }^{1}$, Liang-Dar Hwang ${ }^{4}$, Douglas F. Easton $^{5}$, Paul P. Pharoah ${ }^{5}$, Andrew Berchuck ${ }^{6}$, Linda E. Kelemen ${ }^{7}$, Keitaro Matsuo ${ }^{8}$, Georgia ChenevixTrench $^{1}$, Per Hall ${ }^{3}$, Stig E. Bojesen ${ }^{9,10,11}$, Penelope M Webb ${ }^{1}$ and Stuart MacGregor ${ }^{1}$.

\section{Affiliations:}

${ }^{1}$ QIMR Berghofer Medical Research Institute, Brisbane, QLD, Australia.

${ }^{2}$ Faculty of Medicine, University of Queensland, Brisbane, QLD, Australia.

${ }^{3}$ Medical Epidemiology and Biostatistics, Karolinska Institute, Sweden.

${ }^{4}$ University of Queensland Diamantina Institute, University of Queensland, Translational Research Institute, Brisbane, Queensland, Australia

${ }^{5}$ The Centre for Cancer Genetic Epidemiology, Department of Oncology, University of Cambridge, Cambridge, UK.

${ }^{6}$ Duke Cancer Institute, Duke University Medical Center, Durham, North Carolina, USA.

${ }^{7}$ Departments of Obstetrics and Gynecology and Public Health Sciences, College of Medicine and Hollings Cancer Center, Medical University of South Carolina, Charleston, South Carolina, USA.

${ }^{8}$ Division of Molecular and Clinical Epidemiology, Aichi Cancer Center Research Institute, Nagoya, Japan.

${ }^{9}$ Faculty of Health and Medical Science, University of Copenhagen, DK-2200 Copenhagen, Denmark

${ }^{10}$ Department of Clinical Biochemistry, Herlev and Gentofte Hospital, Copenhagen University Hospital, DK-2730 Herlev, Denmark.

${ }^{11}$ The Copenhagen General Population Study, Herlev and Gentofte Hospital, Copenhagen University Hospital, DK-2730 Herlev, Denmark.

Running title: Does alcohol increase risk of breast and ovarian cancer

Keywords: breast cancer, ovarian cancer, alcohol intake, causal inference, mendelian randomization

\section{Novelty and Impact:}

Using a combination of large scale observational data from three European population-based cohorts and Mendelian randomization analyses on two of the largest cancer consortia, our observational and genetic-derived findings combined reveal that alcohol consumption is unlikely to be a major risk factor for both breast and ovarian cancer susceptibility. 
Correspondence: Jue-Sheng Ong, Level 7 Bancroft, QIMR Berghofer Medical Research Institute, 300 Herston Road, QLD 4006, Herston, Brisbane, Australia. Email: juesheng.ong@qimrberghofer.edu.au Tel: +61738453563

Word count: 4132

Number of tables and figures: 5 Figures

\section{Abbreviations:}

$\mathrm{ADH}$ - Alcohol dehydrogenase

BCAC - Breast Cancer Association Consortium

CCHS - Copenhagen City Heart Study

CGPS - Copenhagen General Population Study

EOC - Epithelial ovarian cancer

ER - Estrogen receptor

GWAS - Genome-wide association studies

HR -Hazard ratio

IVW - Inverse variance weighted

KARMA - Karolinska Mammography Project for Risk Prediction of Breast Cancer

LD - Linkage Disequilibrium

MAF - Minor Allele Frequency

MR - Mendelian randomization

MVMR - Multivariable Mendelian randomization

OCAC - Ovarian Cancer Association Consortium

OR - Odds ratio

SNP - Single nucleotide polymorphism

UKB - UK Biobank

WCRF - World Cancer Research Fund 


\begin{abstract}
Alcohol consumption is correlated positively with risk for breast cancer in observational studies, but observational studies are subject to reverse causation and confounding. The association with epithelial ovarian cancer (EOC) is unclear. We performed both observational Cox-regression and two-sample Mendelian randomization (MR) analyses using data from various European cohort studies (observational) and publicly available cancer consortia (MR). These estimates were compared with World Cancer Research Fund (WCRF) findings. In our observational analyses, the multivariable-adjusted hazard ratios (HR) for a one standard drink/day increase was 1.06 (95\% confidence interval; $1.04,1.08)$ for breast cancer and $1.00(0.92,1.08)$ for EOC, both of which were consistent with previous WCRF findings. MR ORs per genetically predicted one standard drink/day increase estimated via 34 SNPs using MR-PRESSO were $1.00(0.93,1.08)$ for breast cancer and 0.95 $(0.85,1.06)$ for EOC. Stratification by EOC subtype or estrogen receptor status in breast cancers made no meaningful difference to the results. For breast cancer, the confidence intervals for the genetically derived estimates include the point-estimate from observational studies so are not inconsistent with a small increase in risk. Our data provide additional evidence that alcohol intake is unlikely to have anything other than a very small effect on risk of EOC.
\end{abstract}




\section{Introduction}

The World Cancer Research Fund (WCRF) concluded that alcohol intake is a probable cause of breast cancer, with an estimated additional risk of $9 \%$ per $10 \mathrm{~g} /$ day increase in consumption of ethanol, but that there is inadequate evidence to evaluate the association with epithelial ovarian cancer (EOC) ${ }^{1,2}$. It is, however, difficult to measure any effect of elevated alcohol consumption from conventional observational data because of the possible confounding issues: alcohol consumption is itself associated with many other lifestyle and socio-economic factors, which may themselves be a risk factor for cancers and are difficult to quantify. Estimates from observational studies may additionally be biased by other mechanisms ${ }^{3}$, including recall bias due to differences in the completeness of the subjective indications of alcohol consumption across case-control status, selection bias ${ }^{4,5}$ against heavy users of alcohol due to the preferential participation of reasonable healthy individuals and reverse causality. The WCRF estimates are based on data from population-based prospective cohort studies, where exposure information is collected before the event of interest occurs. Although cohort studies in general are less likely to suffer from bias, it is still impossible to rule out confounding. Also, such studies typically only measure exposure variables once or a few times, precluding detailed individual modelling of exposures over time ${ }^{6}$. In principle, double blinded randomized trials are the best way to evaluate causality, but such studies are usually logistically cumbersome and may be unethical.

A Mendelian randomization (MR) study, using genetic variants associated with alcohol consumption as an instrument for alcohol consumption, offers a way to test hypotheses of causality, since the genetic variants are less likely to be associated with other known or unknown confounders, and they are not influenced by (pre-)clinical stages of the diseases ${ }^{7}$. Conceptually, MR relies on the random assortment of genetic variants during meiosis to mimic a "natural" randomized trial ${ }^{8}$. However, for MR estimates to make valid inferences on causality, several assumptions have to be met ${ }^{8}$. Typically, 
such a genetic instrument only explains a fraction of the variance of the exposure variable, and therefore MR studies need very large numbers of participants to answer questions of causality. For alcohol consumption, previous MR studies have used the rs1229984 variant (as this SNP is associated with high levels of acetaldehyde and facial flushing ${ }^{9}$ ) as a genetic instrument to evaluate the link between alcohol intake and disease outcomes ${ }^{10-12}$. Two-sample mendelian randomization is an extension of the traditional MR methodology that leverages greater statistical power for MR analyses by utilizing independent summary-based datasets to derive the genetic association on alcohol and cancer outcome separately without the need for individual level data. As larger GWAS have identified more risk loci and GWAS of outcomes of interest have increased in size, power has recently become adequate to support meaningful statistical inference ${ }^{13}$.

Using several large independent population-based prospective cohorts, we first tested whether alcohol intake is associated with risk of breast cancer and EOC via observational analyses, to compare against previous WCRF findings. We then evaluated whether there is genetic evidence to support a causal relationship between the two using a two-sample Mendelian randomization study approach utilizing consortia data from both the Breast Cancer Association Consortium (BCAC) and Ovarian Cancer Association Consortium (OCAC) for breast and ovarian cancer, respectively. Both observational and genetically derived estimates were then used to infer whether there is evidence for a causal relationship between alcohol intake and these cancers.

\section{Materials and Methods}

\section{Overview}

For the large-scale population-based cohorts, we evaluated the observational association between self-reported alcohol consumption and risk of breast and ovarian cancer via Cox-regression analyses. Study-specific hazard ratio (HR) estimates were then combined via a fixed-effect meta-analysis (separately for each cancer). For the genetic causality analyses, we performed a two-sample MR to 
assess whether genetically predicted alcohol consumption is associated with breast/ovarian cancer susceptibility using publicly available consortia data. Instruments for the MR analyses were obtained from an alcohol consumption GWAS performed on the UK Biobank white British participants.

\section{Description of observational cohort studies}

\section{Data from Copenhagen General Population Study (CGPS) and Copenhagen City Heart Study (CCHS)}

The $\mathrm{CGPS}^{14}$ and the $\mathrm{CCHS}^{15}$ are two large prospective general population studies from Denmark. For both studies, residents from Copenhagen were invited to complete a baseline questionnaire and undergo a physical examination. The questionnaire includes the number of alcoholic drinks consumed daily and this was used to derive standard drinks per week (for this study 1 standard drink 12g ethanol). Blood samples were also obtained. In total, 69420 women participated, 60205 from the CGPS (enrolled between 2003 to 2015) and the remaining 9215 from the CCHS (enrolled during four examinations from 1976-78, 1981-83, 1991-94, and 2001-03). A total of 2312 incident breast cancer and 327 EOC cases were identified. Women with diagnosis of breast cancer prior to examination or who had missing information on covariates were excluded from the analysis. All participants gave written informed consent, and both CCHS and CGPS were approved by the Danish ethics committees (H-KF01-144/01 and KF100.2039/91). Full details on the observational HR analysis in the CGPS and CCHS are provided in Supplementary Methods.

Data from the Karolinska Mammography Project for Risk Prediction of Breast Cancer (KARMA).

The KARMA study is a large Swedish breast cancer prospective cohort study comprising 70877 women who attend regular mammographic screening across four hospitals in Sweden ${ }^{16}$. The aim of the project is to identify risk factors for breast cancer such as mammographic density, genetic and lifestyle 
factors. Information on tumour characteristics, such as ER status, was identified through registers. Self-reported alcohol intake (in grams) estimated via questionnaires was standardised into number of standard drinks/week using a nominal conversion scale of $10 \mathrm{~g} /$ standard drink. For our HR analysis, we identified 985 incident breast cancer cases in a cohort of 60903 women with non-missing data on confounders. We did not perform the analysis for EOC due to the limited number of cases ( $n=57)$. See Supplementary Methods for a complete description.

\section{Data from the UK Biobank cohort}

The UK Biobank (UKB) cohort consists of 502000 middle-aged individuals recruited from across the United Kingdom ${ }^{17}$. The UKB study was approved by the North West Multi-Centre Research Ethics Committee (reference number 06/MRE09/65), participants at the time of recruitment gave informed consent to participate in UK Biobank and be followed up, using a signature capture device. 487910 individuals passed initial genetic quality control protocols. We identified 215830 women genetically similar to those of white-British ancestry through ancestral principal component techniques ${ }^{18}$. The UKB records extensive $(n>2$ 000) phenotypes including anthropometric traits, disease status and lifestyle behaviours. Number of standard drinks per day (one standard drink in the UKBB is roughly equivalent to $12 \mathrm{~g} /$ day of alcohol) was calculated as a weighted sum of daily consumption on various types of alcoholic beverages (Supplementary methods; see also Supplementary Table 1 and 2). Nondrinkers were given a score of zero standard drinks per day. Information about cancer diagnosis among the UKB participants was obtained through data-linkage between self-report, hospital records and cancer registries. Individual cancer types were defined based on ICD-10 definitions, as per previous work ${ }^{19}$. After excluding women with a history of cancer (excluding non-melanoma skin cancer) prior to enrolment including recurrent cancer cases, the cohort comprised 141071 white British women with 4,068 women diagnosed with breast cancer and 425 with EOC. However, the proportion of individuals with missing data on the necessary covariates (e.g. menopausal status, 
education attainment, nulliparity) were relatively high (i.e. only 1771 breast cancer cases and 187 EOC cases had complete data). Cox regression was used to obtain hazard ratios for cancer risk per standard drinks/day increase in alcohol consumption. A complete description of the observational HR analysis for alcohol and cancer in the UKB is provided in Supplementary Methods.

\section{Meta-analysis of observational findings}

We then performed an observational meta-analysis of the association estimates combining the UKB results with those obtained from the CCHS+CGPS and KARMA study for breast cancer and EOC. All association estimates were scaled to reflect a one standard drink per day increase (an increase of $\sim 10 \mathrm{~g} /$ day of ethanol) to facilitate comparison with our MR findings. Estimates were combined under a fixed-effect inverse variance weighted model using the rmeta $\mathrm{R}$ library. These results were then compared against the existing WCRF findings on both cancers ${ }^{1,2}$.

\section{Genetic analyses}

In this two-sample MR study, we derived instrumental variables for alcohol consumption from the UK Biobank cohort. We then evaluated whether these alcohol-associated instruments were associated with breast/ovarian cancer risk using GWAS summary statistics obtained from the Breast Cancer Association Consortium (BCAC) and the Ovarian Cancer Association Consortium (OCAC). A flowchart illustrating the complete MR procedure is shown in Figure 1.

(Figure 1 here)

\section{Breast and ovarian cancer risk GWAS}

The BCAC breast cancer GWAS summary statistics ${ }^{20}$, derived from a total of 122977 cases and 105 974 controls of European ancestries, were obtained from a publicly available repository 
(http://bcac.ccge.medschl.cam.ac.uk/bcacdata/oncoarray). Among these, 69501 of the cases were identified to have ER+ breast cancer, and 21468 breast cancer cases were ER-. Participants in the $B C A C$ were recruited from various case-control and cohort studies around the world. BCAC participants involved in the breast cancer GWAS were genotyped via one of these genotyping platforms: (i) custom Illumina iSelect genotyping arrays, (ii) OncoArray or (iii) the iCOGS array. Genotypes were then imputed against the 1000 Genomes Project Phase III reference panels using IMPUTE2 ${ }^{21}$. A full description of the genetic quality control procedures is given elsewhere ${ }^{20}$. The association between SNPs and cancer outcome were estimated using conventional multiple logistic regression adjusting for top ancestral principal components and age ${ }^{20}$.

The OCAC EOC GWAS summary statistics ${ }^{22}$, derived from a total of 22406 cases and 40941 controls of European ancestries, were obtained from a publicly available repository (http://ocac.ccge.medschl.cam.ac.uk/). The genotyping platforms used were broadly similar to those used in the BCAC breast cancer GWAS. Top ancestral principal components were fitted as covariates in both the breast cancer and EOC GWAS model to account for the presence of population substructure. Prior to our main analyses, we excluded SNPs that were poorly imputed $($ INFO<0.6) or had very low minor allele frequencies $(\mathrm{MAF}<0.01)$ for both GWAS datasets. Similarly, the association between SNPs and cancer outcome were estimated using conventional multiple logistic regression adjusting for top ancestral principal components and age ${ }^{22}$.

\section{Deriving genetic instruments for alcohol consumption (UKB data)}

The complete description of how estimated standard drinks per week was derived via self-reported consumption of alcoholic beverages is provided in Supplementary Methods. In brief, we computed the participants' total alcohol standard drinks per week using both frequency and quantity of alcohol consumption, summing across the alcohol content (in std drinks) from self-reported quantities of 
various types of alcoholic beverage consumed weekly. Non-drinkers are included in the GWAS, and assigned a score of zero standard drinks per week. We performed a GWAS on standard alcoholic drinks per week to calibrate genetic instruments that are predictive of self-reported alcohol consumption among white British women in the UKB. We used the software BOLT-LMM ${ }^{23}$, a Bayesian linear mixed model GWAS framework to explicitly model the genetic relatedness within the sample. Genetic sex, age and 10 ancestral principal components were included as covariates. We performed 2 separate GWAS: using i) the estimated alcohol quantity in both sexes ( $n=432178)$ and ii) the estimated alcohol quantity in females only ( $n=197$ 948). For each alcohol GWAS result, only SNPs that were genome-wide significant and had MAF>0.01 were retained. Clumping on the SNPs were then performed based on LD $\left(r^{2}<0.01\right)$ and maximum distance of $1000 \mathrm{~kb}$ apart to ensure that selected instruments are strictly independent. We identified 72 instruments (including SNP rs1229984) from the combined-sex drinks/week GWAS (Supplementary Table 3). The combined-sex GWAS was used to robustly identify alcohol associated SNPs but in our main MR analysis we adopted SNP effect sizes estimated among females only. In order to ensure that our analyses were protected against weak instrument bias, we only used 34 out of 72 SNPs that were successfully replicated in the female-only alcohol GWAS ( $p<1 e-5$ in females).

\section{Two-sample Mendelian randomization}

GWAS summary statistics were used to obtain association estimates for genetic predictors of alcohol on cancer outcomes (breast or ovarian cancer) from the respective consortia (BCAC and OCAC). The estimated statistical power to detect MR associations at various effect sizes (ORs) is shown in Supplementary Table 4. We extracted the SNP-cancer association estimates and minor allele frequency information for each of the 34 alcohol-associated SNP instruments. We fitted a multiplicative random effect inverse variance weighted (IVW) model to obtain a combined estimate of the causal effect inferred via multiple SNPs ${ }^{24}$. For each test, palindromic SNPs with strands that 
could not be inferred via allele frequency were excluded. The global Cochran $Q$ statistics was first evaluated to determine MR findings with heterogeneous effect sizes. For tests with strong evidence of causal effect heterogeneity, we then repeated our IVW MR analyses by manually filtering out SNPS that showed strong evidence for having heterogenous effect estimates, defined by SNPs with a Cochran' Q statistics exceeding 3.84. The heterogeneity-adjusted MR estimate will be reported in the main results section for traits that have inflated global Cochran $Q$ statistics ( $p$-heterogeneity $<0.05)$.

Previous studies have shown that SNPs associated with alcohol intake might be pleiotropically linked with changes in adiposity, or simply markers for smoking behaviour or education attainment, some of which might confound the association with these cancers. Discarding SNPs on the basis of a pleiotropic association might result in loss of power if the association is linked through the same causal pathway (vertical pleiotropy). However, determining the modes of pleiotropy for each SNP instrument is not a trivial task. Instead, we applied a multivariable MR (MVMR) model to evaluate the direct effect between genetically predicted alcohol intake on breast/ovarian cancer by regressing out the genetic effect of these variants on BMI, BMI-adjusted waist-to-hip ratio, education attainment and cigarettes smoked per day. The marginal OR for alcohol intake (drink/day) on cancer risk(s) after adjusting for the aforementioned risk factors were reported. Curation of the phenotypes used in the MVMR framework is described in Supplementary Methods.

We scaled our MR estimate to reflect a genetically predicted one drink/day increase in alcohol consumption (by multiplying the predicted change in $\log (\mathrm{OR})$ of cancer for 1 standard drink/week by 7). All statistical analyses (including MR sensitivity analyses) were performed in statistical package $R$ using the TwoSampleMR library implemented in the MR-Base platform ${ }^{25}$. 


\section{Sensitivity analyses}

Observational. We assessed evidence for a non-linear relationship between alcohol and breast cancer or EOC outcomes by evaluating the dose-response relationship over strata of increasing alcohol intake in the observational analyses. For the KARMA study (where ER status was available for breast cancer cases), we performed stratified analyses to evaluate whether the alcohol-breast cancer association differed by ER status. There is no information available for ER-status in both the UK Biobank and Copenhagen cohort studies. Due to the high rate of missingness in the covariate data in our UKB (multivariable adjusted) observational HR analyses, we finally performed missing data imputation to evaluate whether the associations varied with more recovered samples (see Supplementary Methods).

$M R$ analyses For the genetically derived estimates, we first attempt to re-evaluate our MR findings using only the rs1229984 SNP as an instrument fitted through a Wald-type estimator ${ }^{26}$. Next we ensure that our findings were not biased by violation of the MR assumptions by repeating our analyses using the following alternative MR models: MR Egger regression ${ }^{27}$, weighted median ${ }^{28}$ and the penalised weighted median model ${ }^{29}$. Deviations of the MR Egger regression intercept from the null for each tested outcome were used to assess evidence of directional pleiotropy. The multi-SNP MR analyses were also repeated using the MR-PRESSO technique ${ }^{30}$ which provides adjusted causal estimates after filtering out heterogeneous SNP-outliers. MR estimates derived using these models were reported alongside the main (IVW) MR results to ensure robustness of findings as different techniques relax different assumptions. Funnel plots and leave-one-out MR plots were also generated to evaluate whether the causal estimates were driven by strong outliers. We also performed a SNPlookup on the recently published alcohol drinks/week GWAS summary data to evaluate whether our instrument-alcohol associations replicate well in the much larger GSCAN alcohol GWAS ${ }^{31}$. Detailed descriptions of the MR sensitivity analyses are provided in the Supplementary Methods. 
For the MR analysis on breast cancer, we additionally performed stratified analyses based on estrogen receptor (ER) status, whilst for EOC, we subsequently evaluated the association of alcohol across different histotypes including the most common HGSOC histotype.

\section{Results}

\section{Conventional observational analyses on alcohol consumption}

\section{Observational association between alcohol consumption with breast and ovarian cancer risk}

Breast cancer. Alcohol consumption was associated with increased risk of breast cancer in the CCHS+CGPS cohorts with a HR of 1.09 per standard drink/day (95\% C.I. 1.05, 1.13), and the Swedish KARMA study with HR $1.07(0.97,1.19)$ while the HR in the UKB dataset was lower (HR $1.04(1.01$, 1.07)). Meta-analysing all these estimates yielded an HR of $1.06(1.04,1.08)$ for risk of breast cancer per one standard drink/day (Figure 2 upper panel)

Ovarian cancer. In the UK Biobank, higher alcohol consumption was associated with a reduction in risk for cancers in the ovary with an age-adjusted HR of $0.92(0.85,0.99)$. Using the multivariable adjusted model ( $N=61,267, N=187$ cases), the $\log (H R)$ was unchanged, albeit with wider confidence intervals (adjusted HR $0.92(0.83,1.03)$ ) due to missing information on covariates. In CCHS+CGPS, the estimated $\mathrm{HR}(\mathrm{HR}=1.07(0.96,1.20))$ was in the opposite direction, but with 95\% Cls that overlapped the estimates from the UKBB. Combining both these estimates yielded an meta-analysed HR of $1.00(0.92,1.08)$ for the risk of EOC per one standard drink of $10 \mathrm{~g}$ alcohol per day increase in alcohol consumption (Figure 2 lower panel).

(Figure 2 here)

Instrumental variable analyses - Genetically predicted alcohol consumption

Association of genetically predicted alcohol consumption with breast cancer and EOC 
(Figure 3 here)

For a one unit increase in genetically predicted daily alcohol intake (using 34 variants), the odds ratio on breast cancer was $1.03(0.93,1.14)$ in standard IVW analysis, with a tighter confidence interval when MR-PRESSO was used to discard one heterogeneous SNPs (OR $1.00[0.93,1.08]$, figure 3). For EOC, the point estimate was less than one, although with relatively wide confidence intervals (OR 0.89 $(0.73,1.08))$. The MR-PRESSO MR OR estimate for EOC was attenuated slightly towards the null (OR $0.95[0.85,1.06])$. Given that all but one of the SNPs (rs62055546) used in our genetic instrument appeared homogeneous in our MR-PRESSO analysis we adopted the MR-PRESSO results as our primary results. Estimates of the MR association under alternative models are shown together in Figure 3. The comparison of our genetically derived estimate against our new observational findings and the WCRF results for breast cancer and EOC risk is provided in Figure 4. The original results not manually filtered for heterogenous SNPs are shown in Supplementary Table 11.

For the multivariable analyses, the estimated marginal OR on breast cancer for one drinks/week increase is $1.03(0.97,1.10)$ in the MVMR model after excluding SNPs with high heterogeneity scores ( $Q>3.84$ ), showing no evidence of effect size attenuation in the univariate MR model due to negative pleiotropy. The MVMR OR estimate for ovarian cancer was $0.97(0.87,1.09)$, see Supplementary Table 12.

(Figure 4 here)

\section{Sensitivity analyses}

The observational HR association between alcohol and breast cancer and EOC for different levels of alcohol consumption indicated no strong evidence for a non-linear relationship (Supplementary Table 5-6). There was limited evidence that the alcohol-breast cancer association differed by ER status in the KARMA study (Supplementary Table 7). Furthermore, the age-adjusted and fully-adjusted models 
gave similar estimates suggesting minimal evidence for confounding on the factors that were controlled for (Supplementary Table 8). To address the high rate of missingness in the UK Biobank multivariable-adjusted HR analyses, multiple imputation was performed to recover missing information in the covariates. We first verified that missingness of our covariates of interests can be predicted by our set of auxiliary variables and other covariates (satisfying the MAR assumption for accurate imputation) (see Supplementary Methods 8; Supplementary Figure 5 and 6). We generated a total of five sets of imputation datasets and pooled the estimates from each individual imputed dataset. The pooled regression estimates from imputed data revealed minor attenuation of the estimate between alcohol drinks/day and both cancers (HR on breast cancer $=1.02[1.00,1.03]$; ovarian cancer $=0.94[0.90,0.99])$ towards the null, providing more precise estimates upon modelling the covariates that previously had high rates of missingness adequately. However, these revised estimates were not meaningfully different from the original multivariable HR estimates as shown by the overlapping confidence intervals (Supplementary Table 9).

(Figure 5 here)

The confidence interval of the estimate from our single SNP MR analyses using the rs1229984 (ADH1B) variant (strongest instrument, explaining $0.23 \%$ of variation in alcohol intake, $p=1 e-128$ ) largely overlaps those of the multi-instrument MR results (Supplementary Figure 1). The MR scatter plots for both cancers using the original 34 alcohol SNP instruments are shown in Figure 5. The F-statistics for our instruments suggest that each of our 34 SNPs are strong instruments, with SNP-alcohol associations (female only) being replicated successfully in the subsequent GSCAN GWAS revealing limited evidence for weak instrument bias (Supplementary Table 13-14). Our Steiger Z-test (Supplementary Table 15) also indicated no evidence for instrument mis-specification (i.e. our SNP instrument $r^{\wedge} 2$ on alcohol $>>r^{\wedge} 2$ on outcome) in our study design. Estimates derived from alternative MR methods (before/after filtering heterogenous instruments) reveal that our findings were robust against weak violation of MR assumption, with the MR-Egger intercepts showing no evidence for 
directional pleiotropy (Supplementary Table 10, 11, 15 and 16). In our pleiotropy assessment, we did not observe evidence for an association between our genetic instruments with potential confounders including age at menarche, oral contraceptive use, smoking quantity, coffee consumption and psychiatric traits, except for BMI in the UKB (See Supplementary Table 17 and 18). However, the magnitude of association between rs1229984 and BMI is so small that it is very unlikely to have substantially biased our estimates. Moreover, our MR-PRESSO findings were statistically consistent with the IVW estimates for each trait. The distribution of effect sizes around the null across multiple sensitivity MR analyses provide strong support for an overall null or a very weak positive relationship between alcohol and breast cancer or EOC.

In our exploratory MR analyses, stratification by ER status produced essentially unchanged the MR results for breast cancer (Supplementary Table 19). Similarly, for subtypes of EOC, results were indistinguishable from those for overall EOC: the high-grade serous estimate was $0.95[0.85,1.06]$ (Supplementary Figure 2).

\section{Discussion}

In this study, we evaluated the association between alcohol consumption and breast and ovarian cancer using conventional observational prospective designs and MR approaches. The point estimates for breast cancer from the observational findings were slightly higher than those from MR, but with overlapping confidence intervals. Although the confidence intervals are wider for the MR estimates, the MR design is likely to be robust to some of the issues which can hamper interpretation of observational studies, such as confounding. Taken together, although our MR estimate overlaps the null, the confidence interval from our MR estimate remains consistent with a modest increase as consistently reported in observational findings. For EOC, the effect appears null in both the observational and MR analyses. 


\section{Comparison with previous literature}

Earlier molecular investigations found that alcohol may be implicated in the development of breast cancer, especially ER+ breast cancer, as it modulates estrogen levels. This adverse influence of alcohol is supported by a study investigating the link between alcohol intake and percentage of breast density (PBD), postulating a potential relationship between alcohol intake and breast cancer susceptibility via increased PBD ${ }^{32}$. Similarly, many observational findings have found that alcohol consumption is associated with risk of breast cancers ${ }^{33-36}$. Results from a large meta-analysis of 27 cohort studies showed that even light drinking ( $<1$ drink/day) is associated with increased risk of breast cancer in women ${ }^{37}$. In our study, we found suggestive evidence from our observational study meta-analysis that increased alcohol consumption is associated with susceptibility for breast cancers, although the magnitude of association was slightly lower than those reported by the WCRF ${ }^{2}$. Here we add MR analysis to provide additional evidence as to whether the association seen in the observational studies represents a true causal association. Whilst the MR estimates had confidence intervals overlapping those from the observational studies, the most likely causal effect was zero (point estimate from MR analysis), with the relatively narrow MR confidence intervals suggesting the causal effect of alcohol intake is at most very small.

The null association between alcohol and EOC was previously shown in the study by Kelemen et al. ${ }^{38}$ pooling together data from 12 case-control studies in OCAC, , and in other pooled case-control ${ }^{39}$ and cohort ${ }^{40}$ studies. In contrast, Cook et al. ${ }^{41}$ showed that self-reported wine consumption was associated with a reduction in EOC risk in a recent Canadian study. One possible explanation for such an association is that the relationship may have been driven by residual confounding with other exposures correlated with socio-economic factors such as educational attainment ${ }^{38,41}$. While the estimated direction of effect for alcohol and ovarian cancer differed (non-significantly) for the UKB 
and Copenhagen cohorts, it is difficult to draw any definitive statement given the overlapping Cls and low number of cases. In our observational meta-analyses, we did not find strong evidence to support a protective association between alcohol and overall EOC, consistent with the WCRF findings ${ }^{1}$. Our MR results for EOC were concordant with the observational study results.

\section{Strength and limitations}

Our large sample size combining data from various sources allow us to assess the role of alcohol consumption on breast/ovarian cancer with reasonably good precision. The MR approach provides additional evidence to triangulate evidence for causality. Our additional MR analysis using alcohol consumption instruments calibrated only among European women helps protect against biased inferences due to weak instruments ${ }^{42}$. While these SNPs combined explain only a small amount ( 0.92\%) of variation in alcohol consumption among women (Supplementary Table 3), due to the large sample sizes from both OCAC and BCAC, the confidence intervals on our MR estimates are reasonably precise. The use of a larger set of SNP instruments also enabled better assessment of potential bias in MR findings through the use of alternative MR models which allow inference under a range of different assumptions (heterogeneity of effect sizes, horizontal pleiotropy).

This study had some limitations. While genetically derived estimates are unlikely to be affected by confounding, the magnitude of association between these genetic instruments and estimated standard drinks detected in GWAS analyses relies on the accuracy of self-reported data, which may contain self-report bias. This might also apply to the UKBB with known healthy volunteer bias ${ }^{43}$, in which the genetic instruments were derived. In recent years, investigators have used multi-instrument MR experiments due to availability of genetic data on large cohorts. The multi-instrument approach is expected to minimise the standard errors around the causal estimates (relative to just a single SNP), although in practice we only found this to be the case when one heterogeneous SNP was discarded 
using MR-PRESSO (Figure 3). Furthermore, the strength of our instruments (explaining $\sim 1 \%$ of variance) remain insufficient to detect small ORs and make further inference on causality, evident from the large degree of overlap in Cls between the observational and MR estimates. The presence of weak instruments in the multi-instrument MR can potentially bias the overall causal estimates towards the null ${ }^{42}$, however this is unlikely the case given that our instruments satisfy the strong instrument criteria (median F-stat 22.2; Interquartile range: 19.5 - 30.3) and most of our variants show evidence of replication in the recent combined-sex drinks/week GWAS from GSCAN ${ }^{31}$. Of the 98 associations reported in the GSCAN alcohol GWAS meta-analysis ${ }^{31}$, 95 replicated in our combined-sex alcohol GWAS in UKBB alone, suggesting that the genotype-alcohol associations used in this study are fairly stable. Results from alternative MR estimators that are robust against horizontal pleiotropy were similar to the IVW findings, although the confidence intervals were wider for those techniques. In contrast, assessing the rs1229984-only estimate remains informative because rs 1229984 by itself is by far the strongest and most extensively studied instrument among the SNP set with well-studied biological insights to justify its association with alcohol consumption. Apart from the ADH1B variant, the biological pathways linking the SNP instruments with alcohol consumption are not well understood; for a trait like alcohol, unmeasured pleiotropy remains a concern as variants might consequently influence alcohol intake through changes in socio-economic status, cultural factors and other social behaviours. In our assessment for bias, the MR-Egger intercept for alcohol-EOC did not show any evidence of directional pleiotropy affecting our MR findings (Supplementary Table 16).

Earlier studies have suggested a link between acetaldehyde (ADH) and cancer cell growth ${ }^{44,45}$, but it is unclear whether these associations were mainly driven by a change in alcohol consumption. Disentangling the complex effects of the rs 1229984 variant is difficult, as previous studies have shown that the variant is associated with esophageal carcinoma ${ }^{46}$, potentially due to accumulation of acetaldehyde among minor allele carriers although our single SNP MR analyses using the rs1229984 
$(A D H 1 B)$ variant find weak evidence for an association with breast cancer risk. Our PheWAS findings on rs 1229984 found no strong evidence that the $A D H 1 B$ instrument was associated with potential risk factors linked with breast or ovarian cancer that are unlikely to be mediated through increased alcohol consumption (Supplementary Table 17). However, we cannot exclude the possibility of rs1229984 being associated with other factors related to carcinogenesis and unmeasured confounders. We are unable to assess whether our MR causal inference remain consistent when we conservatively excluded rs1229984 from the main analyses, as it resulted in wide confidence intervals on the estimate (rs1229984 being the instrument that explains the highest amount of genetic variance, Supplementary Table 3).

For our observational analyses, selection bias might be present for the study cohorts if participants are more healthy than non-participants. Our reliance of self-reported consumption data for the observational analyses is vulnerable to recall error, and the definition of standard drinks may differ slightly across regions, contributing to higher heterogeneity in our exposures. In the covariateadjusted model, the power to detect meaningful associations were hindered by a large degree of missingness on information for the covariates, especially the UK Biobank cohort where the number of cancer events from the multivariable-adjusted analyses were essentially halved. Our multiple imputation analyses recovered valuable information on both smoking pack/years and duration of hormone-replacement therapy and in the pooled regression estimate adjusted for these covariates in the imputed dataset there was a small degree of attenuation of the effect size between alcohol and both cancers (towards the null). Whilst the regression analysis on the imputed dataset characterises confounding better, the estimates based on imputed data (with twice the number of events) were not meaningfully different to the original multivariable adjusted findings for the UKB (Supplementary Table 9) and our conclusions remain essentially unchanged. 
When we compare the observational findings across different cohorts, the breast cancer estimates for one standard drink/day increase from the Copenhagen cohorts and KARMA were broadly consistent with the overwhelming evidence from previous studies. However the estimated HR from the UKB cohort is slightly lower (HR 1.04). This might be explained by the healthy-volunteer selection bias in the recruitment for the UKB cohort, resulting in under-estimation of the true effect size. Conversely, genetic estimates are conceivably less affected by these biases, but they can be vulnerable to biases in the presence of horizontal pleiotropy. We performed sensitivity analyses based on filtering out SNPs with heterogeneous causal effects to reduce the chances of horizontal pleiotropy biasing the estimate including the use of MVMR to adjust for confounding risk factors, although in practice this made no meaningful changes to our results. If participants in the genetic study under-reported their true alcohol consumption this may lead to an underestimate of the SNP-alcohol effect sizes, resulting in an inflated estimate from the MR analysis. If the SNP-alcohol effect sizes were underestimated, it would suggest more strongly that alcohol intake is not causally associated with breast cancer. Finally, our risk estimates from the observational and MR analyses were on similar but not identical scales (i.e. OR versus HR); however for low prevalence outcomes any discrepancy is likely to be small. Individuals with high alcohol consumption are less likely to participate in the studies included, and competing risks after study participation may have influenced our estimates of the effect of risk factor on outcome, but since the observational studies largely reproduced results from other observational studies, this bias is likely to have been small.

The MR estimates for alcohol and risk of breast cancer or EOC remain valid under the assumption that alcohol consumption and $\log (\mathrm{OR})$ of these disease outcomes have a linear relationship. This is a strong assumption, given previous speculation about a J-shaped relationship between alcohol and other disease outcomes (e.g. cardiovascular diseases) where abstainers are at higher risk similar to those drinking more than moderate amounts ${ }^{47,48}$. Despite our inability to perform MR-by-stratum 
(evaluating effect of genetically predicted alcohol consumption on risk of disease at various consumption levels) due to insufficient sample size, our observational findings show little evidence that the relationship between alcohol intake and these cancers is non-linear. Given that the alcohol variants (such as rs1229984) might predict both drinker status and quantity consumed, modelling the MR association within drinkers-only might potentially induce collider bias ${ }^{49}$.

Taken together, Mendelian randomization analyses are not inconsistent with findings from several cohort studies showing that moderate alcohol consumption is associated with a modest increase in risk of breast cancer (upper $95 \% \mathrm{Cl}$ of OR on breast cancer being 1.10 for a genetically predicted 1 drink per day increase). For EOC alcohol intake is unlikely to have anything other than a very small effect on risk.

\section{Additional information:}

\section{Ethics statement:}

The UK Biobank study was approved by the National Research Ethics Service Committee North West-Haydock, whereby all study procedures were performed in accordance with the World Medical Association Declaration of Helsinki ethical principles for medical research. The CCHS and CGPS studies were approved by the Herlev and Gentofte Hospital and by a Danish ethical committee (H-KF-01-144/01), and was conducted according to the Declaration of Helsinki. Finally, the KARMA study was approved by the ethical board at the Karolinska Institute.

\section{Funding:}

This work was conducted using the UK Biobank Resource (application number 25331). This work is supported by a project grant (1123248) from the Australian National Health and Medical Research Council (NHMRC). Jue-Sheng Ong received scholarship support from the University of Queensland and QIMR Berghofer Medical Research Institute. Stuart MacGregor, Penelope M. Webb and Georgia Chenevix-Trench are supported by fellowships from the NHMRC. Funding for the CCHS and CGPS were obtained from the Chief Physician Johan Boserup and Lise Boserup Fund, the Danish Medical 
Research Council and Herlev Hospital.

\section{Authorship:}

SM, EMD, PMW, GCT, SEB and PH designed the study and obtained funding. JSO performed the UKB analyses. ME provided data for the KARMA study cohort. SEB provided data for the Copenhagen City Heart Study and Copenhagen General Population Study. JSO, JA, EMD, ME, SEB and SM analysed the data. JSO, SM, SEB, PMW and GCT wrote the first draft of the paper. LEK, KM, PP, AB, DFE and LDH provided critical feedback on the manuscript. All authors contributed to the final version of the paper.

\section{Acknowledgement:}

We would like to thank the staff and participants of the CCHS, CGPS, KARMA and UK Biobank cohort study. We thank Scott Wood and John Pearson from QIMR Berghofer for IT support. The breast cancer genome-wide association analyses were supported by the Government of Canada through Genome Canada and the Canadian Institutes of Health Research, the 'Ministère de l'Économie, de la Science et de l'Innovation du Québec' through Genome Québec and grant PSR-SIIRI-701, The National Institutes of Health (U19 CA148065, X01HG007492), Cancer Research UK (C1287/A10118, C1287/A16563, C1287/A10710) and The European Union (HEALTH-F2-2009-223175 and H2020 633784 and 634935). All studies and funders are listed in Michailidou et al (2017). The Ovarian Cancer Association Consortium is supported by a grant from the Ovarian Cancer Research Fund thanks to donations by the family and friends of Kathryn Sladek Smith (PPD/RPCI.07). The scientific development and funding for this project were in part supported by the US National Cancer Institute GAME-ON Post-GWAS Initiative (U19-CA148112). This study made use of data generated by the Wellcome Trust Case Control consortium that was funded by the Wellcome Trust under award 076113. The results published here are in part based upon data generated by The Cancer Genome Atlas Pilot Project established by the National Cancer Institute and National Human Genome Research Institute (dbGap accession number phs000178.v8.p7). Detailed funding and acknowledgements for the BCAC and OCAC studies are provided in the supplementary material.

\section{Conflict of interest:}

The authors declare no conflict of interest.

\section{Data availability statement:}


Individual level genetic and phenotypic data of UK Biobank participants are available at http://biobank.ctsu.ox.ac.uk through formal application. GWAS meta-analyses data for BCAC and OCAC were publically available and downloaded from the corresponding consortium sites. The authors declare that summary statistics data supporting the findings of this study are available within the paper and its supplementary information files.

\section{References}

1. World Cancer Research Fund. Systematic Literature Review Continuous Update Project Report: The Associations between Food, Nutrition and Physical Activity and the Risk of Ovarian Cancer [Internet]. 2013 [cited 2019 May 15];Available from: https://www.wcrf.org/sites/default/files/ovarian-cancer-slr.pdf

2. World Cancer Research Fund. Continuous Update Project Expert Report: Diet, nutrition, physical activity and cancer [Internet]. 2018 [cited 2019 May 15];Available from: dietandcancerreport.org

3. Boyko EJ. Observational research--opportunities and limitations. J Diabetes Complications 2013;27:642-8.

4. Naimi TS, Stockwell T, Zhao J, Xuan Z, Dangardt F, Saitz R, Liang W, Chikritzhs T. Selection biases in observational studies affect associations between "moderate" alcohol consumption and mortality. Addiction 2017;112:207-14.

5. Gmel G. Beneficial effects of moderate alcohol use-a case for Occam's razor? Addiction2017;112:215-7.

6. Rossow I, Norström T. The use of epidemiology in alcohol research. Addiction 2013;108:20-5.

7. Palmer TM, Lawlor DA, Harbord RM, Sheehan NA, Tobias JH, Timpson NJ, Davey Smith G, Sterne JAC. Using multiple genetic variants as instrumental variables for modifiable risk factors. Stat Methods Med Res 2012;21:223-42.

8. Thanassoulis G, O'Donnell CJ. Mendelian randomization: nature's randomized trial in the postgenome era. JAMA2009;301:2386-8.

9. Macgregor S, Lind PA, Bucholz KK, Hansell NK, Madden PAF, Richter MM, Montgomery GW, Martin NG, Heath AC, Whitfield JB. Associations of ADH and ALDH2 gene variation with self report alcohol reactions, consumption and dependence: an integrated analysis. Hum Mol Genet 2009;18:580-93.

10. Skaaby $T$, Kilpeläinen TO, Taylor AE, Mahendran $\mathrm{Y}$, Wong A, Ahluwalia TS, Paternoster L, 
Trompet S, Stott DJ, Flexeder C, Zhou A, Brusselle G, et al. Association of alcohol consumption with allergic disease and asthma: a multicenter Mendelian Randomization analysis. Addiction [Internet] 2018;Available from: http://dx.doi.org/10.1111/add.14438

11. Pierce BL, Kraft $P$, Zhang C. Mendelian randomization studies of cancer risk: a literature review. Curr Epidemiol Rep 2018;5:184-96.

12. Holmes MV, Dale CE, Zuccolo L, Silverwood RJ, Guo Y, Ye Z, Prieto-Merino D, Dehghan A, Trompet S, Wong A, Cavadino A, Drogan D, et al. Association between alcohol and cardiovascular disease: Mendelian randomisation analysis based on individual participant data. BMJ 2014;349:g4164.

13. Burgess S, Malarstig A. Using Mendelian randomization to assess and develop clinical interventions: limitations and benefits. J Comp Eff Res 2013;2:209-12.

14. Näslund-Koch C, Nordestgaard BG, Bojesen SE. Increased Risk for Other Cancers in Addition to Breast Cancer for CHEK2*1100delC Heterozygotes Estimated From the Copenhagen General Population Study. J Clin Oncol 2016;34:1208-16.

15. Kodal JB, Kobylecki CJ, Vedel-Krogh S, Nordestgaard BG, Bojesen SE. hypomethylation, lung function, lung function decline and respiratory symptoms. Eur Respir J [Internet] 2018;51. Available from: http://dx.doi.org/10.1183/13993003.01512-2017

16. Gabrielson M, Eriksson M, Hammarström M, Borgquist S, Leifland K, Czene K, Hall P. Cohort Profile: The Karolinska Mammography Project for Risk Prediction of Breast Cancer (KARMA). Int J Epidemiol 2017;46:1740-1g.

17. Bycroft C, Freeman C, Petkova D, Band G, Elliott LT, Sharp K, Motyer A, Vukcevic D, Delaneau O, O'Connell J, Cortes A, Welsh S, et al. The UK Biobank resource with deep phenotyping and genomic data. Nature 2018;562:203-9.

18. MacGregor S, Ong J-S, An J, Han X, Zhou T, Siggs OM, Law MH, Souzeau E, Sharma S, Lynn DJ, Beesley J, Sheldrick B, et al. Genome-wide association study of intraocular pressure uncovers new pathways to glaucoma. Nat Genet 2018;50:1067-71.

19. Ong J-S, An J, Law MH, Whiteman DC, Neale RE, Gharahkhani P, MacGregor S. Height and overall cancer risk and mortality: evidence from a Mendelian randomisation study on 310,000 UK Biobank participants. Br J Cancer 2018;118:1262-7.

20. Michailidou K, Lindström S, Dennis J, Beesley J, Hui S, Kar S, Lemaçon A, Soucy P, Glubb D, Rostamianfar A, Bolla MK, Wang Q, et al. Association analysis identifies 65 new breast cancer risk loci. Nature 2017;551:92-4.

21. Howie B, Fuchsberger C, Stephens M, Marchini J, Abecasis GR. Fast and accurate genotype imputation in genome-wide association studies through pre-phasing. Nat Genet 2012;44:955-9.

22. Phelan CM, Kuchenbaecker KB, Tyrer JP, Kar SP, Lawrenson K, Winham SJ, Dennis J, Pirie A, Riggan MJ, Chornokur G, Earp MA, Lyra PC Jr, et al. Identification of 12 new susceptibility loci for different histotypes of epithelial ovarian cancer. Nat Genet 2017;49:680-91.

23. Loh P-R, Tucker G, Bulik-Sullivan BK, Vilhjálmsson BJ, Finucane HK, Salem RM, Chasman DI, Ridker PM, Neale BM, Berger B, Patterson N, Price AL. Efficient Bayesian mixed-model analysis increases association power in large cohorts. Nat Genet 2015;47:284-90. 
24. Burgess S, Small DS, Thompson SG. A review of instrumental variable estimators for Mendelian randomization. Stat Methods Med Res 2017;26:2333-55.

25. Hemani G, Zheng J, Elsworth B, Wade KH, Haberland V, Baird D, Laurin C, Burgess S, Bowden J, Langdon R, Tan VY, Yarmolinsky J, et al. The MR-Base platform supports systematic causal inference across the human phenome. Elife [Internet] 2018;7. Available from: http://dx.doi.org/10.7554/eLife.34408

26. Burgess S, Scott RA, Timpson NJ, Davey Smith G, Thompson SG, EPIC- InterAct Consortium. Using published data in Mendelian randomization: a blueprint for efficient identification of causal risk factors. Eur J Epidemiol 2015;30:543-52.

27. Bowden J, Davey Smith G, Burgess S. Mendelian randomization with invalid instruments: effect estimation and bias detection through Egger regression. Int J Epidemiol 2015;44:512-25.

28. Bowden J, Davey Smith G, Haycock PC, Burgess S. Consistent Estimation in Mendelian Randomization with Some Invalid Instruments Using a Weighted Median Estimator. Genet Epidemiol 2016;40:304-14.

29. Burgess S, Dudbridge F, Thompson SG. Combining information on multiple instrumental variables in Mendelian randomization: comparison of allele score and summarized data methods. Stat Med 2016;35:1880-906.

30. Verbanck M, Chen C-Y, Neale B, Do R. Detection of widespread horizontal pleiotropy in causal relationships inferred from Mendelian randomization between complex traits and diseases. Nat Genet 2018;50:693-8.

31. Liu M, Jiang Y, Wedow R, Li Y, Brazel DM, Chen F, Datta G, Davila-Velderrain J, McGuire D, Tian C, Zhan X, 23andMe Research Team, et al. Association studies of up to 1.2 million individuals yield new insights into the genetic etiology of tobacco and alcohol use. Nat Genet 2019;51:23744.

32. Ziembicki S, Zhu J, Tse E, Martin L, Minkin S, Boyd NF. The Association between Alcohol Consumption and Breast Density: A Systematic Review and Meta-analysis. Cancer Epidemiol Biomarkers Prev 2017;26:170-8.

33. Romieu I, Scoccianti C, Chajès V, de Batlle J, Biessy C, Dossus L, Baglietto L, Clavel-Chapelon F, Overvad K, Olsen A, Tjønneland A, Kaaks R, et al. Alcohol intake and breast cancer in the European prospective investigation into cancer and nutrition. Int J Cancer 2015;137:1921-30.

34. Chen WY, Rosner B, Hankinson SE, Colditz GA, Willett WC. Moderate alcohol consumption during adult life, drinking patterns, and breast cancer risk. JAMA 2011;306:1884-90.

35. Li Cl, Chlebowski RT, Freiberg M, Johnson KC, Kuller L, Lane D, Lessin L, O'Sullivan MJ, Wactawski-Wende J, Yasmeen S, Prentice R. Alcohol consumption and risk of postmenopausal breast cancer by subtype: the women's health initiative observational study. J Natl Cancer Inst 2010;102:1422-31.

36. Zakhari S, Hoek JB. Epidemiology of Moderate Alcohol Consumption and Breast Cancer: Association or Causation? Cancers [Internet] 2018;10. Available from: http://dx.doi.org/10.3390/cancers10100349

37. Choi Y-J, Myung S-K, Lee J-H. Light Alcohol Drinking and Risk of Cancer: A Meta-Analysis of Cohort Studies. Cancer Res Treat 2018;50:474-87. 
38. Kelemen LE, Bandera EV, Terry KL, Rossing MA, Brinton LA, Doherty JA, Ness RB, Kjaer SK, Chang-Claude J, Köbel M, Lurie G, Thompson PJ, et al. Recent alcohol consumption and risk of incident ovarian carcinoma: a pooled analysis of 5,342 cases and 10,358 controls from the Ovarian Cancer Association Consortium. BMC Cancer 2013;13:28.

39. Rota M, Pasquali E, Scotti L, Pelucchi C, Tramacere I, Islami F, Negri E, Boffetta P, Bellocco R, Corrao G, La Vecchia C, Bagnardi V. Alcohol drinking and epithelial ovarian cancer risk. a systematic review and meta-analysis. Gynecol Oncol 2012;125:758-63.

40. Genkinger JM, Hunter DJ, Spiegelman D, Anderson KE, Buring JE, Freudenheim JL, Goldbohm RA, Harnack L, Hankinson SE, Larsson SC, Leitzmann M, McCullough ML, et al. Alcohol intake and ovarian cancer risk: a pooled analysis of 10 cohort studies. Br J Cancer 2006;94:757-62.

41. Cook LS, Leung ACY, Swenerton K, Gallagher RP, Magliocco A, Steed H, Koebel M, Nation J, Eshragh S, Brooks-Wilson A, Le ND. Adult lifetime alcohol consumption and invasive epithelial ovarian cancer risk in a population-based case-control study. Gynecol Oncol 2016;140:277-84.

42. Burgess S, Davies NM, Thompson SG. Bias due to participant overlap in two-sample Mendelian randomization. Genet Epidemiol 2016;40:597-608.

43. Fry A, Littlejohns TJ, Sudlow C, Doherty N, Adamska L, Sprosen T, Collins R, Allen NE. Comparison of Sociodemographic and Health-Related Characteristics of UK Biobank Participants With Those of the General Population. Am J Epidemiol 2017;186:1026-34.

44. Seitz HK, Becker P. Alcohol metabolism and cancer risk. Alcohol Res Health 2007;30:38-41, 447.

45. Seitz HK, Stickel F. Acetaldehyde as an underestimated risk factor for cancer development: role of genetics in ethanol metabolism. Genes Nutr 2010;5:121-8.

46. Gu H, Gong D, Ding G, Zhang W, Liu C, Jiang P, Chen S, Chen Y. A variant allele of ADH1B and ALDH2, is associated with the risk of esophageal cancer. Exp Ther Med 2012;4:135-40.

47. Zhao J, Stockwell T, Roemer A, Naimi T, Chikritzhs T. Alcohol Consumption and Mortality From Coronary Heart Disease: An Updated Meta-Analysis of Cohort Studies. J Stud Alcohol Drugs 2017;78:375-86.

48. Stockwell T, Zhao J, Chikritzhs T. Whether Low Volume Alcohol Use Is Cardio-Protective Is Important for Public Health Policy So the Available Evidence Deserves Critical Analysis: The Authors Respond. J. Stud. Alcohol Drugs2017;78:392-3.

49. Munafò MR, Tilling K, Taylor AE, Evans DM, Davey Smith G. Collider scope: when selection bias can substantially influence observed associations. Int J Epidemiol 2018;47:226-35. 


\section{Figure Legends}

Figure 1. Schematic diagram illustrating the Mendelian randomization (MR) framework for the main analysis.

Figure $\mathbf{2}$ Meta-analysis of the observational hazard ratio estimates for daily alcohol consumption on breast and ovarian cancer. Estimates were adjusted for BMI, oral contraceptive use, nulliparity, physical activity and education attainment. Please refer to supplementary table 7 for the estimated HR adjusted for age only. There is no strong evidence for effect heterogeneity $(p>0.1)$ among estimates from each of these studies.

Figure 3. Mendelian randomization estimates for the relationship between alcohol consumption and risk of breast/ovarian cancers. The confidence interval around the estimates narrowed down after we removed SNPs via the outlier test in MR-PRESSO.

Figure 4. Comparison of observational and genetic (MR) estimates for the association between standard drink per day with breast and ovarian cancer risk. Observational HR estimates were obtained via fixed effect meta-analysis of the studies used in the main analysis. The MR-PRESSO outlier-adjusted estimates were reported here as the MR-analysis findings.

Figure 5. Scatter plot for the genetic association between alcohol drinks/week SNP instruments and risk of breast and ovarian cancers. The slope of the fitted line in the scatter plots reflect the MR causal estimates for each type of MR estimator. The scatter plot shows the association of a genetically predicted one standard drinks/week increase on $\log (\mathrm{OR})$ of the outcome (cancer) risk inferred via each alcohol SNP instrument. The panel (A) refers to the plot for overall EOC; (B) refers to the plot for the risk of overall breast. For both plots, the right-most point refers to the rs 1229984 SNP estimate. The forest plot for the individual SNP estimates along with the leave-one-SNP-out MR forest plot is shown in Supplementary Figure 3 and 4. 


\title{
Supplementary Material for manuscript "Evaluating the role of alcohol consumption in breast and ovarian cancer susceptibility using population-based cohort studies and two-sample Mendelian randomization analyses"
}

\author{
Authors \\ Jue-Sheng Ong*, Eske M. Derks, Mikael Eriksson, Jiyuan An, Liang-Dar Hwang, Douglas F. Easton, Paul P. \\ Bojesen, Penelope M Webb and Stuart MacGregor.

\footnotetext{
*Correspondending author. To request additional information, please email

juesheng.ong@qimrberghofer.edu.au or stuart.macgregor@qimrberghofer.edu.au
} Pharoah, Andrew Berchuck, Linda E. Kelemen, Keitaro Matsuo, Georgia Chenevix-Trench, Per Hall, Stig E.

\section{Table of Contents}

Detailed Funding and Acknowledgement for OCAC and BCAC 4

Supplementary materials and methods 10

Method 1. Estimating phenotypic variance tagged by genetic variants 10

Method 2. Deriving number of alcoholic standard drinks in UK Biobank 10

Method 3. Observational data for the Copenhagen General Population Study and the Copenhagen

City Heart Study 11

Method 4. Observational data for the Karolinska Mammography Project for Risk Prediction of Breast

Cancer (KARMA). 11

Method 5. Modelling the association between alcohol and BrCa/OvCa in UK Biobank 11

Method 6. Sensitivity analyses for the Mendelian randomization study 12

Method 7. Multivariable Mendelian randomization analyses 12

Method 8. Imputation of missing covariate data in UK Biobank observational analyses 13

Supplementary tables 17

Supplementary Table 1. Conversion table for various alcoholic beverage into units of standard drinks.

Supplementary Table 2. Estimated average of total standard drinks per week across each consumption category among women in the UK Biobank cohort.

Supplementary Table 3. Phenotypic variance (weekly alcohol intake) explained by alcohol-associated SNP instruments used in MR analysis. $\quad 18$

Supplementary Table 4. Power calculation for MR analysis 21 
Supplementary Table 5. Estimated hazard ratios for the association between daily alcohol consumption and breast and ovarian cancer in the CCHS+CGPS for different consumption levels. $\quad 22$

Supplementary Table 6. Estimated hazard ratio for the association between daily alcohol drinks with breast cancer in the KARMA study for different consumption levels.

Supplementary Table 7. Estimated hazard ratio for the association between daily alcohol drinks with breast cancer in the KARMA study stratified by ER status of breast cancer.

Supplementary Table 8. Comparison of age- and fully-adjusted hazard ratios for the association between daily alcohol intake and breast and ovarian cancer risk for each study.

Supplementary Table 9. Comparison of pooled HR estimates obtained through missing covariate data imputation against original estimates in the UK Biobank breast and ovarian cancer cohort. $\quad 25$

Supplementary Table 10. Global test for heterogeneity on MR causal estimates.

Supplementary Table 11. Comparison of MR estimates across alternative MR methods before and after filtering for SNP-heterogeneity

Supplementary Table 12. Multivariable MR analysis adjusting for the effect on adiposity, smoking behaviour and education attainment

Supplementary Table 13. Comparison of instrument strength across various sets of instrument from UK Biobank drinks/week GWAS

Supplementary Table 14. Evidence of replication of UKBB estimated drinks/week (females only) SNPassociation in the GSCAN drinks/week GWAS summary statistics 30

Supplementary Table 15. MR Steiger Z-test for directionality.

Supplementary Table 16. Estimate of the MR-Egger intercept for the MR analysis between alcohol intake with breast and ovarian cancer risk.

Supplementary Table 17. Pleiotropy assessment on ADH1B SNP rs1229984 using the online Phenoscanner database

Supplementary Table 18. Pleiotropy assessment on ADH1B SNP rs1229984 using database GeneATLAS.

Supplementary Table 19. MR association between standard drink/day alcohol consumption and breast cancer risk stratified by ER status.

Supplementary Figure 1. Comparison of MR association between estimated one standard drink/day and breast cancer and EOC susceptibility using single instrument and multiple instrument approaches

Supplementary Figure 2. MR association between estimated one standard drink/day and EOC susceptibility based on EOC subtypes.

Supplementary Figure 3. Scatter plot and forest plot for the genetic association between alcohol drinks/week SNP instruments and risk of breast and ovarian cancers 
Supplementary Figure 4. MR Leave-one-out plots for the genetic association between alcohol drinks/week SNP instruments and risk of breast and ovarian cancers.

Supplementary Figure 5. Phenotypic correlation between covariates and auxilliary variables used in the multiple imputation analysis for the UKB cohort.

Supplementary Figure 6. Indicator matrix on the predictability of covariates with missing data from other covariates and auxiliary variables estimated from MiCE 


\section{Detailed Funding and Acknowledgement for OCAC and BCAC}

The breast cancer genome-wide association analyses were supported by the Government of Canada through Genome Canada and the Canadian Institutes of Health Research, the 'Ministère de l'Économie, de la Science et de I'Innovation du Québec' through Genome Québec and grant PSR-SIIRI-701, The National Institutes of Health (U19 CA148065, X01HG007492), Cancer Research UK (C1287/A10118, C1287/A16563, C1287/A10710) and The European Union (HEALTH-F2-2009-223175 and H2020 633784 and 634935). All studies and funders are listed in Michailidou et al (2017)(1).

The Ovarian Cancer Association Consortium is supported by a grant from the Ovarian Cancer Research Fund thanks to donations by the family and friends of Kathryn Sladek Smith (PPD/RPCl.07). The scientific development and funding for this project were in part supported by the US National Cancer Institute GAME-ON Post-GWAS Initiative (U19-CA148112). This study made use of data generated by the Wellcome Trust Case Control consortium that was funded by the Wellcome Trust under award 076113. The results published here are in part based upon data generated by The Cancer Genome Atlas Pilot Project established by the National Cancer Institute and National Human Genome Research Institute (dbGap accession number phs000178.v8.p7). The OCAC OncoArray genotyping project was funded through grants from the U.S. National Institutes of Health (CA1X01HG007491-01 (C.I.A.), U19-CA148112 (T.A.S.), R01-CA149429 (C.M.P.) and R01-CA058598 (M.T.G.); Canadian Institutes of Health Research (MOP-86727 (L.E.K.) and the Ovarian Cancer Research Fund (A.B.). The COGS project was funded through a European Commission's Seventh Framework Programme grant (agreement number 223175 - HEALTHF2-2009-223175).

Funding for individual studies: AAS: National Institutes of Health (RO1-CA142081); AOV: The Canadian Institutes for Health Research (MOP-86727); AUS: The Australian Ovarian Cancer Study Group was supported by the U.S. Army Medical Research and Materiel Command (DAMD17-01-1-0729), National Health \& Medical Research Council of Australia (199600, 400413 and 400281), Cancer Councils of New South Wales, Victoria, Queensland, South Australia and Tasmania and Cancer Foundation of Western Australia (Multi-State Applications 191, 211 and 182). The Australian Ovarian Cancer Study gratefully acknowledges additional support from Ovarian Cancer Australia and the Peter MacCallum Foundation; BAV: ELAN Funds of the University of Erlangen-Nuremberg; BEL: National Kankerplan; BGS: Breast Cancer Now, Institute of Cancer Research; BVU: Vanderbilt University Medical Center's BioVU is supported by the 1S10RR025141-01 instrumentation award and Vanderbilt CTSA grant from the National Institutes of Health (NIH)/National Center for Advancing Translational Sciences (NCATS) (ULTR000445); CAM: National Institutes of Health Research Cambridge Biomedical Research Centre and Cancer Research UK Cambridge Cancer Centre; CHA: Innovative Research Team in University (PCSIRT) in China (IRT1076); CNI: Instituto de Salud Carlos III (PI 12/01319); Ministerio de Economía y Competitividad (SAF2012); COE: Department of Defense (W81XWH-11-2-0131); CON: National Institutes of Health (R01-CA063678, R01-CA074850; R01CA080742); DKE: Ovarian Cancer Research Fund; DOV: National Institutes of Health R01-CA112523 and R01-CA87538; EMC: Dutch Cancer Society (EMC 2014-6699); EPC: The coordination of EPIC is financially 
supported by the European Commission (DG-SANCO) and the International Agency for Research on Cancer. The national cohorts are supported by Danish Cancer Society (Denmark) (EMC 2014-6699); Ligue Contre le Cancer, Institut Gustave Roussy, Mutuelle Générale de l'Education Nationale, Institut National de la Santé et de la Recherche Médicale (INSERM) (France); German Cancer Aid, German Cancer Research Center (DKFZ), Federal Ministry of Education and Research (BMBF) (Germany); the Hellenic Health Foundation (Greece); Associazione Italiana per la Ricerca sul Cancro-AIRC-Italy and National Research Council (Italy); Dutch Ministry of Public Health, Welfare and Sports (VWS), Netherlands Cancer Registry (NKR), LK Research Funds, Dutch Prevention Funds, Dutch ZON (Zorg Onderzoek Nederland), World Cancer Research Fund (WCRF), Statistics Netherlands (The Netherlands); ERC-2009-AdG 232997 and Nordforsk, Nordic Centre of Excellence programme on Food, Nutrition and Health (Norway); Health Research Fund (FIS), PI13/00061 to Granada, PI13/01162 to EPIC-Murcia, Regional Governments of Andalucía, Asturias, Basque Country, Murcia and Navarra, ISCIII RETIC (RD06/0020) (Spain); Swedish Cancer Society, Swedish Research Council and County Councils of Skåne and Västerbotten (Sweden); Cancer Research UK (14136 to EPIC-Norfolk; C570/A16491 and C8221/A19170 to EPIC-Oxford), Medical Research Council (1000143 to EPIC-Norfolk, MR/M012190/1 to EPIC-Oxford) (United Kingdom); GER: German Federal Ministry of Education and Research, Programme of Clinical Biomedical Research (01 GB 9401) and the German Cancer Research Center (DKFZ); GRC: This research has been co-financed by the European Union (European Social Fund - ESF) and Greek national funds through the Operational Program "Education and Lifelong Learning" of the National Strategic Reference Framework (NSRF) - Research Funding Program of the General Secretariat for Research \& Technology: SYN11_10_19 NBCA. Investing in knowledge society through the European Social Fund; GRR: Roswell Park Cancer Institute Alliance Foundation, P30 CA016056; HAW: U.S. National Institutes of Health (R01-CA58598, N01-CN-55424 and N01-PC-67001); HJO: Intramural funding; Rudolf-Bartling Foundation; HMO: Intramural funding; RudolfBartling Foundation; HOC: Helsinki University Research Fund; HOP: University of Pittsburgh School of Medicine Dean's Faculty Advancement Award (F. Modugno), Department of Defense (DAMD17-02-10669) and NCl (K07-CA080668, R01-CA95023, P50-CA159981 MO1-RR000056 R01-CA126841); HUO: Intramural funding; Rudolf-Bartling Foundation; JGO: JSPS KAKENHI grant; JPN: Grant-in-Aid for the Third Term Comprehensive 10-Year Strategy for Cancer Control from the Ministry of Health, Labour and Welfare; KRA: This study (Ko-EVE) was supported by a grant from the Korea Health Technology R\&D Project through the Korea Health Industry Development Institute (KHIDI), and the National R\&D Program for Cancer Control, Ministry of Health \& Welfare, Republic of Korea (HI16C1127; 0920010); LAX: American Cancer Society Early Detection Professorship (SIOP-06-258-01-COUN) and the National Center for Advancing Translational Sciences (NCATS), Grant UL1TR000124; LUN: ERC-2011-AdG 294576-risk factors cancer, Swedish Cancer Society, Swedish Research Council, Beta Kamprad Foundation; MAC: National Institutes of Health (R01-CA122443, P30-CA15083, P50-CA136393); Mayo Foundation; Minnesota Ovarian Cancer Alliance; Fred C. and Katherine B. Andersen Foundation; Fraternal Order of Eagles; MAL: Funding for this study was provided by research grant R01- CA61107 from the National Cancer Institute, Bethesda, MD, research grant 9422252 from the Danish Cancer Society, Copenhagen, Denmark; and the Mermaid I project; MAS: Malaysian Ministry of Higher Education (UM.C/HIR/MOHE/06) and Cancer Research Initiatives Foundation; MAY: National Institutes of Health (R01-CA122443, P30-CA15083, P50-CA136393); Mayo Foundation; Minnesota Ovarian Cancer Alliance; Fred C. and Katherine B. Andersen Foundation; MCC: MCCS cohort recruitment was funded by VicHealth and Cancer Council Victoria. Cancer Council 
Victoria, National Health and Medical Research Council of Australia (NHMRC) grants number 209057, 251533, 396414, and 504715; MDA: DOD Ovarian Cancer Research Program (W81XWH-07-0449); MEC: NIH (CA54281, CA164973, CA63464); MOF: Moffitt Cancer Center, Merck Pharmaceuticals, the state of Florida, Hillsborough County, and the city of Tampa; NCO: National Institutes of Health (R01-CA76016) and the Department of Defense (DAMD17-02-1-0666); NEC: National Institutes of Health R01-CA54419 and P50-CA105009 and Department of Defense W81XWH-10-1-02802; NHS: UM1 CA186107, P01 CA87969, R01 CA49449, R01-CA67262, UM1 CA176726; NJO: National Cancer Institute (NIH-K07 CA095666, R01-CA83918, NIH-K22-CA138563, and P30-CA072720) and the Cancer Institute of New Jersey; If Sara Olson and/or Irene Orlow is a co-author, please add NCI CCSG award (P30-CA008748) to the funding sources; NOR: Helse Vest, The Norwegian Cancer Society, The Research Council of Norway; NTH: Radboud University Medical Centre; OPL: National Health and Medical Research Council (NHMRC) of Australia (APP1025142) and Brisbane Women's Club; ORE: OHSU Foundation; OVA: This work was supported by Canadian Institutes of Health Research grant (MOP-86727) and by NIH/NCI 1 R01CA160669-01A1; PLC: Intramural Research Program of the National Cancer Institute; POC: Pomeranian Medical University; POL: Intramural Research Program of the National Cancer Institute; PVD: Canadian Cancer Society and Cancer Research Society GRePEC Program; RBH: National Health and Medical Research Council of Australia; RMH: Cancer Research UK, Royal Marsden Hospital; RPC: National Institute of Health (P50 CA159981, R01CA126841); SEA: Cancer Research UK (C490/A10119 C490/A10124); UK National Institute for Health Research Biomedical Research Centres at the University of Cambridge; SIS: The Sister Study (SISTER) is supported by the Intramural Research Program of the NIH, National Institute of Environmental Health Sciences (Z01-ES044005 and Z01-ES049033); SMC: The Swedish Research Council; SON: National Health Research and Development Program, Health Canada, grant 6613-1415-53; SRO: Cancer Research UK (C536/A13086, C536/A6689) and Imperial Experimental Cancer Research Centre (C1312/A15589); STA: NIH grants U01 CA71966 and U01 CA69417; SWE: Swedish Cancer foundation, WeCanCureCancer and årKampMotCancer foundation; SWH: NIH (NCI) grant R37-CA070867; TBO: National Institutes of Health (R01-CA106414-A2), American Cancer Society (CRTG-00-196-01-CCE), Department of Defense (DAMD1798-1-8659), Celma Mastery Ovarian Cancer Foundation; TOR: NIH grants R01 CA063678 and R01 CA063682; UCl: NIH R01-CA058860 and the Lon V Smith Foundation grant LVS-39420; UHN: Princess Margaret Cancer Centre Foundation-Bridge for the Cure; UKO: The UKOPS study was funded by The Eve Appeal (The Oak Foundation) and supported by the National Institute for Health Research University College London Hospitals Biomedical Research Centre; UKR: Cancer Research UK (C490/A6187), UK National Institute for Health Research Biomedical Research Centres at the University of Cambridge; USC: P01CA17054, P30CA14089, R01CA61132, N01PC67010, R03CA113148, R03CA115195, N01CN025403, and California Cancer Research Program (00-01389V-20170, 2II0200); VAN: BC Cancer Foundation, VGH \& UBC Hospital Foundation; VTL: NIH K05-CA154337; WMH: National Health and Medical Research Council of Australia, Enabling Grants ID 310670 \& ID 628903. Cancer Institute NSW Grants 12/RIG/1-17 \& 15/RIG/1-16; WOC: National Science Centre (N N301 5645 40), The Maria Sklodowska-Curie Memorial Cancer Centre and Institute of Oncology, Warsaw, Poland. 


\section{Australian Ovarian Cancer Study (AOCS) Group}

D. Bowtell, D. Gertig, A. Green, P. Parsons, N. Hayward, D. Whiteman, P. M. Webb, A. C. Green, N. K. Hayward, P. G. Parsons, D. M. Purdie, B. M. Smithers, D. Gotley, A. Clouston, I. Brown, S. Moore. K. Harrap, T. Sadkowski, S. O'Brien, E. Minehan, D. Roffe, S. O'Keefe, S. Lipshut, G. Connor, H. Berry, F. Walker, T. Barnes, J. Thomas, L. Terry, M. Connard, L. Bowes, M-R. Malt, J. White, C. Mosse, N. Tait, C. Bambach, A. Biankan, R. Brancatisano, M. Coleman , M. Cox , S. Deane , G. L. Falk , J. Gallagher, M. Hollands , T. Hugh , D. Hunt , J. Jorgensen, C. Martin, M. Richardson, G. Smith , R. Smith , D. Storey , J. Avramovic , J. Croese, J. D'Arcy , S. Fairley, J. Hansen, J. Masson, L. Nathanson, B. O'Loughlin , L. Rutherford, R. Turner , M. Windsor, J. Bessell , P. Devitt, G. Jamieson, D. Watson, S. Blamey, A. Boussioutas, R. Cade , G. Crosthwaite, I. Faragher , J. Gribbin , G. Hebbard, G. Kiroff , B. Mann , R. Millar , P. O'Brien , R. Thomas ,

S. Wood , S. Archer, K. Faulkner, J. Hamdorf (AOCS); R. Stuart-Harris, F. Kirsten, J. Rutovitz, P. Clingan, A .Glasgow, A. Proietto, S. Braye, G. Otton, J. Shannon, T. Bonaventura, J. Stewart, S. Begbie, M. Friedlander, D. Bell, S. Baron-Hay, G. Gard, D. Nevell, N. Pavlakis, S. Valmadre, B. Young, C. Camaris, R. Crouch, L. Edwards, N. Hacker, D. Marsden, G. Robertson, P. Beale, J. Beith, J. Carter, C. Dalrymple, R. Houghton, P. Russell, L. Anderson, M. Links, J. Grygiel, J. Hill, A. Brand, K. Byth, R. Jaworski, P. Harnett, R. Sharma, .G Wain, D. Purdie, D.C. Whiteman, B. Ward, D. Papadimos, A. Crandon, M. Cummings, K. Horwood. A. Obermair, L. Perrin, D. Wyld, J. Nicklin, M. Davy, M. K. Oehler, C. Hall, T. Dodd, T. Healy, K. Pittman, D. Henderson, J. Miller, J. Pierdes, A. Achan, P. Blomfield, D. Challis, R. Mclntosh, A. Parker, B. Brown, R. Rome, D. Allen, P. Grant, S. Hyde, R. Laurie, M. Robbie, D. Healy, T. Jobling, T. Manolitsas, J. McNealage, P Rogers, B. Susil, E. Sumithran, I. Simpson, I. Haviv, K. Phillips, D. Rischin, S. Fox, D. Johnson, S. Lade, P. Waring, M. Loughrey, N .O'Callaghan, B. Murray, L. Mileshkin, P. Allan; V. Billson, J. Pyman, D. Neesham, M. Quinn, A. Hamilton, C. Underhill, R. Bell, L. F Ng, R. Blum, V .Ganju, I. Hammond, C. Stewart, Y. Leung, M. Buck, N. Zeps

\section{Ovarian Cancer Prognosis and Lifestyle (OPAL) Group}

A. Obermair, M. Friedlander, P. Grant

\section{Acknowledgements}

We are grateful to the family and friends of Kathryn Sladek Smith for their generous support of the Ovarian Cancer Association Consortium through their donations to the Ovarian Cancer Research Fund. The OncoArray and COGS genotyping projects would not have been possible without the contributions of the following: Kyriaki Michailidou, Manjeet K. Bolla, Qin Wang (BCAC), Marjorie J. Riggan (OCAC), Rosalind A. Eeles, Ali Amin Al Olama, Zsofia Kote-Jarai, Sara Benlloch (PRACTICAL), Antonis Antoniou, Lesley McGuffog, Fergus Couch and Ken Offit (CIMBA), Joe Dennis, Jonathan P. Tyrer, Siddhartha Kar, Alison M. Dunning, Andrew Lee, and Ed Dicks, Craig Luccarini and the staff of the Centre for Genetic Epidemiology Laboratory, Javier Benitez, Anna Gonzalez-Neira and the staff of the CNIO genotyping unit, Jacques Simard and Daniel C. Tessier, Francois Bacot, Daniel Vincent, Sylvie LaBoissière and Frederic Robidoux and the staff of the McGill University and Génome Québec Innovation Centre, Sune F. Nielsen, Borge G. Nordestgaard, and the staff of the Copenhagen DNA laboratory, and Julie M. Cunningham, Sharon A. Windebank, Christopher A. Hilker, Jeffrey Meyer and the staff of Mayo Clinic Genotyping Core Facility. We pay special tribute to the contribution of Professor Brian Henderson to the GAME-ON consortium; to Olga $M$. Sinilnikova for her contribution to CIMBA and for her part in the initiation and coordination of GEMO 
until she sadly passed away on the 30th June 2014 and to Catherine M. Phelan for her contribution to OCAC and coordination of the OncoArray until she passed away on 22 September 2017. We thank the study participants, doctors, nurses, clinical and scientific collaborators, health care providers and health information sources who have contributed to the many studies contributing to this manuscript.

Acknowledgements for individual studies: AOV: We thank Jennifer Koziak, Mie Konno, Michelle Darago, Faye Chambers and the Tom Baker Cancer Centre Translational Laboratories; AUS: The AOCS also acknowledges the cooperation of the participating institutions in Australia and acknowledges the contribution of the study nurses, research assistants and all clinical and scientific collaborators to the study. The complete AOCS Study Group can be found at www.aocstudy.org. We would like to thank all of the women who participated in these research programs; BEL: We would like to thank Gilian Peuteman, Thomas Van Brussel, Annick Van den Broeck and Joke De Roover for technical assistance; BGS: The Institute of Cancer Research (ICR) acknowledges NHS funding to the NIHR Biomedical Research Centre. We thank the Study staff, study participants, doctors, nurses, health care providers and health information sources who have contributed to the study; BVU: The dataset(s) used for the analyses described were obtained from Vanderbilt University Medical Center's BioVU; CHA: Innovative Research Team in University (PCSIRT) in China (IRT1076); CHN: To thank all members of Department of Obstetrics and Gynaecology, Hebei Medical University, Fourth Hospital and Department of Molecular Biology, Hebei Medical University, Fourth Hospital; COE: Gynecologic Cancer Center of Excellence; CON: The cooperation of the 32 Connecticut hospitals, including Stamford Hospital, in allowing patient access, is gratefully acknowledged. This study was approved by the State of Connecticut Department of Public Health Human Investigation Committee. Certain data used in this study were obtained from the Connecticut Tumor Registry in the Connecticut Department of Public Health. The authors assume full responsibility for analyses and interpretation of these data; EPC: To thank all members and investigators of the Rotterdam Ovarian Cancer Study; GER: The German Ovarian Cancer Study (GER) thank Ursula Eilber for competent technical assistance; MAS: We would like to thank Famida Zulkifli and Ms Moey for assistance in patient recruitment, data collection and sample preparation; MCC: Cases and their vital status were ascertained through the Victorian Cancer Registry (VCR) and the Australian Institute of Health and Welfare (AIHW), including the National Death Index and the Australian Cancer Database; MOF: the Total Cancer Care ${ }^{\mathrm{TM}}$ Protocol and the Collaborative Data Services and Tissue Core Facilities at the H. Lee Moffitt Cancer Center \& Research Institute, an $\mathrm{NCl}$ designated Comprehensive Cancer Center (P30-CA076292), Merck Pharmaceuticals and the state of Florida; NHS: The NHS/NHSII studies thank the following state cancer registries for their help: AL, AZ, AR, CA, CO, CT, DE, FL, GA, ID, IL, IN, IA, KY, LA, ME, MD, MA, MI, NE, NH, NJ, NY, NC, ND, OH, OK, OR, PA, RI, SC, TN, TX, VA, WA, and WY; OPL: Members of the OPAL Study Group (http://opalstudy.qimrberghofer.edu.au/); SEA: SEARCH team, Craig Luccarini, Caroline Baynes, Don Conroy; SRO: To thank all members of Scottish Gynaecological Clinical Trails group and SCOTROC1 investigators; SWE: Swedish Cancer foundation, WeCanCureCancer and årKampMotCancer foundation; SWH: We thank the participants and the research staff of the Shanghai Women's Health Study for making this study possible; UHN: Princess Margaret Cancer Centre Foundation-Bridge for the Cure; UKO: We particularly thank I. Jacobs, M.Widschwendter, E. Wozniak, A. Ryan, J. Ford and N. Balogun for their contribution to the study; UKR: Carole Pye; VAN: BC Cancer Foundation, VGH \& UBC Hospital Foundation; 
WMH: We thank the Gynaecological Oncology Biobank at Westmead, a member of the Australasian Biospecimen Network-Oncology group. 


\section{Supplementary materials and methods}

\section{Method 1. Estimating phenotypic variance tagged by genetic variants}

For a set of $k$ SNP instruments and phenotype of interest $Y$, we used the following equation to estimate the total phenotypic variance explained by instruments:

$$
r^{2}=\sum_{i=1}^{k} 2 p_{i}\left(1-p_{i}\right) \beta_{i}^{2} / \operatorname{Var}(Y)
$$

Where $p_{i}$ and $\beta_{i}$ refers to the minor allele frequency and the magnitude of association of the $\mathrm{i}$-th SNP.

\section{Method 2. Deriving number of alcoholic standard drinks in UK Biobank}

Information on quantity and frequency of alcohol consumption, was obtained through self-report questionnaires in the UKB. Frequency of consumption (AC-Frequency) was assessed in 501,718 participants (UKB field IDs: 1558) with the item "About how often do you drink alcohol?". Frequency was originally assessed at a scale ranging from 1 (daily or almost daily) to 6 (never), but was recoded so that a lower score represented less frequent drinking. For individuals who reported multiple instances (via multiple visits) of alcohol intake, only the first assessment was used.

In those who drank at least once or twice a week, information on quantity of consumption (AC-Quantity) was assessed $(n=348,039)$. AC-Quantity was assessed based on the average weekly alcohol intake for five general classes: red wine (1568), champagne plus white wine (1578), spirits (1598), beer plus cider (1558), and fortified wine (1608). The following item was used: "In an average WEEK, how many servings of \{class of alcohol\} would you drink?". To combine the different classes of alcohol, we followed the procedures developed by Clarke et al. (2) with some minor changes, as discussed below. To calculate the total number of alcohol standard drinks, the number of reported drinks was multiplied with a conversion factor depending on the class of alcohol (Supplementary Table 1). For the less-frequent drinkers, we repeated the same procedure using equivalent assessments available for their monthly (instead of weekly) quantity. These values were converted to weekly units by dividing by 4.3 ( 30 days/7). The average value for total drinks/day was then calculated for each frequency category (see Supplementary Table 2). We subsequently identified outliers as those who had a score that deviated $>5$ SD from the average in each female drinker category.

We then imputed the missing values with the sex-specific average of total standard drinks/week for each of the 6 frequency categories. This allowed us to utilise data from the maximum number of female participants for the GWAS analyses, further improving the statistical power to detect robust genetic instruments for alcohol intake. Finally, we selected only individuals of white-British ancestry based on clustering via ancestral principal components and performed the GWAS analyses as per description in 
the main text. Unlike Clarke et al. (2), we did not include weight (in $\mathrm{kg}$ ) as a covariate in the GWAS model.

Method 3. Observational data for the Copenhagen General Population Study and the Copenhagen City Heart Study

We performed cox regression analyses of the 69,420 women to evaluate the relationship between daily number of standard alcohol drinks of $10 \mathrm{~g}$ alcohol per day on breast cancer risk or ovarian cancer risk, adjusted for cohort, age, parity, use of contraceptives, hormone therapy, daily and cumulative tobacco use, height, weight, BMI, nulliparity and menopausal status. Follow up began at date of examination, and ended at next examination, date of first breast or ovarian cancer diagnosis, date of death, or end of follow up (December 31 ${ }^{\text {st }}$ 2016), whichever came first. Each woman from CCHS could contribute with up to 4 observations, depending on the number of examinations of that woman. Recurrent events were not considered. Women with event prior to entry were excluded from that particular analysis. Analyses were performed using STATA 13.1 SE.

\section{Method 4. Observational data for the Karolinska Mammography Project for Risk Prediction of Breast Cancer (KARMA).}

In the KARMA study, alcohol drinking behaviour of the participants was captured through diet questionnaires, along with information on other breast cancer related risk factors (Parity, age at menarche, anthropometric traits, smoking and menopausal status). Self-reported alcohol intake (in grams) estimated via questionnaires was standardised into number of standard drinks/week using a nominal conversion scale of $10 \mathrm{~g} /$ standard drink. All individuals diagnosed with breast/ovarian cancer prior to recruitment were excluded. In total, 60,903 women (985 incident breast cancer cases) with nonmissing data on confounders were used for the KARMA observational analyses. Among the 985 cases, 867 were ER+ while the remaining 118 were ER-. We derived both a crude estimate adjusting for only age, and a multivariable-adjusted estimate accounting for parity, age at menarche, BMI, height, hip circumference, smoking and menopausal status. The Software SAS v9.4 was used for the KARMA observational analyses.

\section{Method 5. Modelling the association between alcohol and BrCa/OvCa in UK Biobank}

Using our derived alcohol intake phenotype (estimated standard drinks per week), we used Cox proportional hazards models to quantify the association between alcohol consumption and breast/ovarian cancer risk. All cancers diagnosed prior to recruitment (prevalent cases) were excluded, retaining only 427 ovarian cancer and 4,081 breast cancer incident cases (cohort size, n=145,089 women) all of which are filtered to be genetically unrelated. The crude model adjusts for only top 10 ancestral principal components, with age at last follow-up as the underlying time variable. The adjusted model (complete_model) additionally included the following covariates: number of live births, cigarette pack years, smoking status (former, current, non-smoker), coffee intake, education attainment, BMI, height, physical activity, age at menarche and menopausal status. Individuals that are cryptically related 
were removed by filtering individual-pairs (and retaining only one of each) with a strong degree of genetic relatedness $(\hat{\pi}>0.2)$ estimated through genetic data. The sample size for the adjusted model were lower due to missing data on some of the covariables (See Supplementary Table 7). The Cox proportional hazard models were implemented using Surv() in R.

\section{Method 6. Sensitivity analyses for the Mendelian randomization study}

Weak violation of the MR assumptions (such as SNP-confounding and invalid instruments) can severely bias MR inferences. To address these issues, we performed sensitivity analyses using alternative MR models (MR Egger, Weighted median, MR-PRESSO) that are least affected by these violations. The MREgger intercepts were computed to evaluate evidence of directional pleiotropy, which would be captured by a non-zero intercept estimate. To generate a reliable causal estimate, we attempted to screen for heterogeneity in MR causal estimates through the global and individual SNP heterogeneity Cochran Q test (Supplementary Table 10). We conservatively excluded any SNPs with a Q-score greater than 3.84 (3). These estimates are provided in Supplementary Table 11. In the main text, we included the MR-PRESSO estimates alongside the IVW estimate to provide an estimate of the MR association that are robust against SNP-heterogeneity. Furthermore, leave-one-out MR analyses (MR estimates leaving out one SNP at a time) were also used to detect outliers in our MR estimates. For the single instrument (rs1229984) MR analyses, the causal estimate was computed using a wald-type ratio estimator. We further performed a phenome-wide association scan (PheWAS) on the rs1229984 SNP, which is the strongest alcohol-associated genetic variant against publicly available GWAS datasets to assess potential pleiotropic association with known confounders. The Steiger-Z test was also used to perform MR directionality test to ensure SNP instruments are acting on the correct hypothesized causal pathway and avoid instrument mis-specification.

For the MR-PRESSO analysis, each trait (cancer outcomes) was performed using the mr_presso() function in the mr-presso $\mathrm{R}$ package (4), with default setting at $\mathrm{Nb}=5000$ iterations, enabled distortion test, enabled outlier test and applied an outlier-significance threshold of 0.05 . SNP alleles were harmonized prior to running these analyses via the harmonize() feature in the MR-Base R-package. All sensitivity analyses apart from the MR-PRESSO tests were performed using the MendelianRandomization and MR-Base R package $(5,6)$.

\section{Method 7. Multivariable Mendelian randomization analyses}

When the exposure of interest is correlated with various other risk factors, MR estimates can be severely biased by horizontal pleiotropy. However, some of the SNP association on these risk factors might manifest in the same causal pathway, where manual removal of these variants would reduce statistical power for MR. The multivariable MR model can hence be used to evaluate the direct effect between our exposure of interest on the outcome while adjusting the genetic effect our SNPs exert on a 
set of risk factors. This is implemented through the mv_multiple() function curated within the TwoSampleMR R package. The risk factors that we adjusted for includes BMI, BMI-adjusted waist-to-hip ratio (WHR), education attainment and cigarette smoked per day, all of which had data available in the UK Biobank cohort. We performed the GWAS for BMI and WHR using identical procedure to those described in previous work (Gharahkhani et al. 2019). We recoded education attainment as an ordinary variable, with the lowest score reflecting lowest attained academic qualification, with individuals that reported to have "Other professional qualification" excluded from the GWAS analysis. The variable is then ranked-transformed to allow effect size to be interpreted in changes in SD units. For cigarette per day, we excluded individuals reported to have smoked more than 99 cigarettes per day but retained individuals that reported zero consumption levels. For both education attainment and cigarette/day, the GWAS was performed on 438,609 white British individuals in the UK Biobank using the BOLT-LMM software, adjusting for recruitment age, sex and top 10 ancestral principal components. The effect estimate of the alcohol instruments on these risk factors obtained directly from the GWAS summary statistics were then applied into the MVMR model.

\section{Method 8. Imputation of missing covariate data in UK Biobank observational analyses}

In our primary cox-regression analyses, participants with missing information on any of the covariates are removed from the analyses. For the UK Biobank cohort, this eventually resulted in a much lower sample size (cases were essentially halved) for the multifactor-adjusted cox regression analyses. To be able to include additional samples, we imputed missing confounders via multiple imputation analyses in R. We extracted a collection of phenotypes on socio-economic status, sexual development, and female sex-specific risk factors (see list below). The pairwise correlation between these phenotypes and the covariates (for imputation) are shown in Supplementary Figure 5.

Finalised Variables considered in the multiple imputation analyses including auxiliary variables (AX): "Ever smoked", "Age at menarche", "Duration of daily moderate physical activity", "Duration of daily vigorous physical activity", "coffee cups/day", "drink temperature (AX) ", "Townsend deprivation index", "Duration of hormone replacement therapy (HRT)", "Ever had HRT", "Menopausal status", "Smoking pack years", "Overall health rating (AX)", "Body mass index", "Frequency of visit from friends and family $(A X)$ ", "Number of live births", "Age at first sexual intercourse (AX)", "Renting or privately owns an apartment $(A X)$ ", "Academic qualifications", "Age completed full time education (AX)", "Number of medications $(A X)$ ", "Number of vehicles in the household $(A X)$ ", "Birth weight (AX)", "Height"

Before we can proceed to impute the missing values on the covariates, we first extracted a series of auxiliary variables from the UK Biobank to assess whether missingness on any of these covariate of interests can be predicted by these auxiliary variables (along with the other covariates with low missingness). This can be done via performing a logistic regression fitting all auxiliary variables against the missingness status of the covariate (i.e. we defined a 'missingness status' phenotype where we recoded missing values as 1 , and non-missing values as 0 for the trait under assessment). We performed this check for each covariate we intend to impute, to help assess plausibility of the Missing-at-random 
(MAR) assumption prior to multiple imputation. In our dataset, the missingness for each of our covariate of interests can be predicted by atleast one auxiliary variable (average predictors $\sim 3$ ), hence there is strong evidence to support the MAR assumption on the pattern of missingness for these covariates. We also compared these predictability estimates against those from the predictor matrix derived from the MiCE initiation process (accessible via imp\$predictorMatrix upon executing "imp=mice $($ dat, $m=1$, max_iter $=0, .)$.$) ". The comparison of the predictability of each covariate and the$ specific models used for the imputation of the trait is summarized in Table M8. We did NOT impute our outcome (cancer diagnosis) and exposure (alcohol drinks/week) phenotype to prevent bias from circular associations - these variables were manually omitted from the multiple imputation process.

The missing data imputation was performed using mice() from the MiCE R package for missing data imputation (available at https://github.com/stefvanbuuren/mice). The process was set for a maximum of 5 iterations, generating 5 distinct datasets with pseudo-random seed set at seed=123. We applied different imputation models to impute covariate data of varying characteristics: the predictive mean matching model $(\mathrm{pmm})$ for quantitative trait, logistic regression (logreg) for binary trait, proportional odds model (plr) for ordered categorical traits and polytomous regression (polyreg) for ordered/unordered categorical traits. Cox proportional hazard estimates from the 5 distinct imputed datasets were then computed using Surv() and then pooled together via the pool() function in MiCE. The pooled estimates were finally compared against the original estimate (multivariable adjusted Coxmodel) for both cancers to evaluate potential difference in observational findings. Note that the multiple imputation analysis was performed on the entire set of UK Biobank female participants used in the main analysis but were evaluated separately for both cancers. 
Table M8. Evaluation of missingness in covariate data in the UK Biobank breast/ovarian cancer cohort

\begin{tabular}{|c|c|c|c|c|c|c|c|c|}
\hline \multirow[t]{2}{*}{ Variable } & \multirow[t]{2}{*}{$\begin{array}{l}\text { Data } \\
\text { structure }\end{array}$} & \multicolumn{2}{|c|}{$\begin{array}{l}\text { Proportion of missing } \\
\text { values }\end{array}$} & \multirow{2}{*}{$\begin{array}{l}\text { Predictable } \\
\text { from auxiliary } \\
\text { variables }\end{array}$} & \multirow{2}{*}{$\begin{array}{l}\text { Number of } \\
\text { predictors } \\
\text { (based on } \\
\text { MiCE } \\
\text { prediction } \\
\text { matrix) }\end{array}$} & \multirow{2}{*}{$\begin{array}{l}\text { Number of } \\
\text { predictors } \\
\text { (based on } \\
\text { missingness } \\
\text { regression) }\end{array}$} & \multirow[t]{2}{*}{$\begin{array}{l}\text { Imputation } \\
\text { model }\end{array}$} & \multirow{2}{*}{$\begin{array}{l}\text { Used } \\
\text { in } \\
\text { analyses }\end{array}$} \\
\hline & & $\begin{array}{l}\text { Breast } \\
\text { cancer UKB } \\
\text { cohort }\end{array}$ & $\begin{array}{l}\text { Ovarian } \\
\text { cancer } \\
\text { UKB } \\
\text { cohort }\end{array}$ & & & & & \\
\hline $\begin{array}{l}\text { Duration } \\
\text { of Hormone } \\
\text { replacement } \\
\text { therapy }\end{array}$ & Continuous & 0.732 & 0.713 & Yes & 3 & 6 & $\mathrm{pmm}$ & $\mathrm{N} / \mathrm{A}$ \\
\hline $\begin{array}{l}\text { Cigarettes } \\
\text { smoked in pack } \\
\text { years }\end{array}$ & Continuous & 0.361 & 0.352 & Yes & 12 & 5 & $\mathrm{pmm}$ & N/A \\
\hline Qualification & $\begin{array}{c}\text { Categorical } \\
\text { (ordered) }\end{array}$ & 0.172 & 0.167 & Yes & 13 & 8 & polr & Yes \\
\hline $\begin{array}{l}\text { Coffee } \\
\text { consumption } \\
\text { (cup/day) }\end{array}$ & Continuous & 0.073 & 0.071 & Yes & 1 & 2 & $\mathrm{pmm}$ & Yes \\
\hline $\begin{array}{l}\text { Duration } \\
\text { of daily } \\
\text { moderate physical } \\
\text { activity }\end{array}$ & $\begin{array}{l}\text { Categorical } \\
\text { (ordered) }\end{array}$ & 0.055 & 0.054 & Yes & 3 & 1 & polr & Yes \\
\hline $\begin{array}{l}\text { Duration } \\
\text { of daily vigorous } \\
\text { physical activity }\end{array}$ & $\begin{array}{l}\text { Categorical } \\
\text { (ordered) }\end{array}$ & 0.051 & 0.049 & Yes & 3 & 2 & polr & Yes \\
\hline $\begin{array}{l}\text { Age } \\
\text { at menarche }\end{array}$ & Continuous & 0.029 & 0.028 & Yes & 1 & 1 & $\mathrm{pmm}$ & Yes \\
\hline $\begin{array}{l}\text { Ever } \\
\text { smoked }\end{array}$ & Binary & 0.003 & 0.003 & Yes & 3 & 3 & logreg & Yes \\
\hline BMI & Continuous & 0.003 & 0.002 & Yes & 11 & 3 & $\mathrm{pmm}$ & Yes \\
\hline Height & Continuous & 0.002 & 0.002 & Yes & 7 & 2 & $\mathrm{pmm}$ & Yes \\
\hline $\begin{array}{l}\text { Menopausal } \\
\text { status }\end{array}$ & $\begin{array}{l}\text { Categorical } \\
\text { (unordered) }\end{array}$ & 0.002 & 0.001 & Yes & 7 & 3 & polyreg & Yes \\
\hline $\begin{array}{l}\text { Number } \\
\text { of live births }\end{array}$ & Continuous & 0.001 & 0.001 & Yes & 8 & 4 & $\mathrm{pmm}$ & Yes \\
\hline $\begin{array}{l}\text { Townsend } \\
\text { deprivation } \\
\text { index }\end{array}$ & Continuous & 0.001 & 0.001 & Yes & 0 & 0 & $\mathrm{pmm}$ & Yes \\
\hline $\begin{array}{l}\text { Participant } \\
\text { ID }\end{array}$ & N/A & 0 & 0 & $\mathrm{~N} / \mathrm{A}$ & $\mathrm{N} / \mathrm{A}$ & $\mathrm{N} / \mathrm{A}$ & N/A & $\begin{array}{l}\text { Reference } \\
\text { only }\end{array}$ \\
\hline Sex & N/A & 0 & 0 & $\mathrm{~N} / \mathrm{A}$ & N/A & $\mathrm{N} / \mathrm{A}$ & N/A & N/A \\
\hline
\end{tabular}

The model "pmm" refers to the predictive mean matching model for quantitative traits, "polr" refers to proportional odds model for ordered categorical variable(s), "polyreg" refers to polytomous logistic regression for unordered categorical 
variable(s) and "logreg" refers to logistic regression for binary coded variable(s). The performance of the logistic regression on missingness is poor (since ncases [missing=1] for traits with very low missingness is very small) apart from duration of HRT and cigarette smoked in pack years; note that these estimates are indicative of plausibility for MAR assumption only, since the multiple imputation process was wholly performed via the MiCE R package. 


\section{Supplementary tables}

Supplementary Table 1. Conversion table for various alcoholic beverage into units of standard drinks.

\begin{tabular}{|l|l|l|}
\hline Type of alcoholic beverage & $\begin{array}{l}\text { UKB-ID for } \\
\text { weekly; monthly }\end{array}$ & Standard drinks equivalence \\
\hline Red wine & $1568 ; 4407$ & 1.67 \\
\hline White wine & $1578 ; 4418$ & 1.67 \\
\hline Fortitude wine & $1608 ; 4451$ & 2.25 \\
\hline Spirits & $1598 ; 4440$ & 1 \\
\hline Pint of beer & $1588 ; 4429$ & 2.3 \\
\hline Other alcoholic drinks & $5364 ; 4462$ & 1.1 \\
\hline
\end{tabular}

Supplementary Table 2. Estimated average of total standard drinks per week across each consumption category among women in the UK Biobank cohort.

\begin{tabular}{|l|l|l|}
\hline Alcohol frequency category & Description & $\begin{array}{l}\text { Average no. of standard } \\
\text { drinks/week }\end{array}$ \\
\hline Category 1 & Daily or almost daily & 12.5 \\
\hline Category 2 & 3 or 4 times a week & 8.0 \\
\hline Category 3 & Once or twice a week & 5.0 \\
\hline Category 4 & 1 or 3 times a month & 0.8 \\
\hline Category 5 & Special occasion only & 0.5 \\
\hline Category 6 & Never & 0 \\
\hline Total & - & 9.4 \\
\hline
\end{tabular}


Supplementary Table 3. Phenotypic variance (weekly alcohol intake) explained by alcohol-associated SNP instruments used in MR analysis. The variance explained by instrument $\left(r^{2}\right)$ was estimated using the formula provided in Supplementary methods. BETA refer to the magnitude of association on estimated standard drinks/week per effect allele (EA) of the SNP. EAF is the frequency of the effect allele. The variance for drinks/week was estimated to be 256.67 (across both sexes) and 114.60 (in females). Only SNPs with $\mathrm{P}<1 \mathrm{e}-5$ in the female-only GWAS are used as instruments for the MR analyses.

\begin{tabular}{|c|c|c|c|c|c|c|c|c|c|c|c|}
\hline \multirow[t]{2}{*}{ SNP } & \multirow[t]{2}{*}{ CHR } & \multirow[t]{2}{*}{ EA } & \multirow[t]{2}{*}{ NEA } & \multicolumn{4}{|c|}{ Estimated across both sexes } & \multicolumn{4}{|c|}{ Estimated in females only } \\
\hline & & & & EAF & BETA & P-value & $r^{\wedge} 2$ & EAF & BETA & P-value & $\mathbf{r}^{\wedge} 2$ \\
\hline rs1229984 & 4 & $\mathrm{~T}$ & $\mathrm{C}$ & 0.02 & -3.421 & $1.10 \mathrm{E}-218$ & 0.0020 & 0.02 & -2.471 & $6.80 \mathrm{E}-121$ & 0.0023 \\
\hline rs1260326 & 2 & $\mathrm{~T}$ & $\mathrm{C}$ & 0.39 & -0.529 & $3.50 \mathrm{E}-58$ & 0.0005 & 0.39 & -0.315 & $4.60 \mathrm{E}-23$ & 0.0004 \\
\hline rs11940694 & 4 & A & G & 0.39 & -0.518 & $9.70 \mathrm{E}-55$ & 0.0005 & 0.39 & -0.342 & $1.40 \mathrm{E}-26$ & 0.0005 \\
\hline rs13107325 & 4 & C & $\mathrm{T}$ & 0.93 & 0.706 & $4.50 \mathrm{E}-30$ & 0.0003 & 0.93 & 0.300 & $6.70 \mathrm{E}-07$ & 0.0001 \\
\hline rs1302808 & 4 & C & $A$ & 0.80 & -0.446 & $1.90 \mathrm{E}-27$ & 0.0002 & 0.80 & -0.318 & $6.50 \mathrm{E}-16$ & 0.0003 \\
\hline rs62055546 & 17 & $A$ & $\mathrm{C}$ & 0.78 & 0.401 & $1.50 \mathrm{E}-24$ & 0.0002 & 0.78 & 0.258 & $1.10 \mathrm{E}-11$ & 0.0002 \\
\hline rs1004787 & 2 & G & $A$ & 0.47 & -0.310 & $9.90 \mathrm{E}-22$ & 0.0002 & 0.47 & -0.235 & $1.10 \mathrm{E}-13$ & 0.0002 \\
\hline rs11604680 & 11 & $A$ & G & 0.68 & 0.305 & 5.50E-19 & 0.0002 & 0.68 & 0.167 & $5.80 \mathrm{E}-07$ & 0.0001 \\
\hline rs56094641 & 16 & $A$ & G & 0.60 & 0.251 & $2.30 \mathrm{E}-14$ & 0.0001 & 0.60 & 0.072 & $2.20 \mathrm{E}-02$ & 0.0000 \\
\hline rs61873510 & 10 & G & $\mathrm{T}$ & 0.67 & 0.263 & $5.70 \mathrm{E}-14$ & 0.0001 & 0.67 & 0.181 & $5.80 \mathrm{E}-08$ & 0.0001 \\
\hline rs9822731 & 3 & $\mathrm{~T}$ & $\mathrm{C}$ & 0.78 & -0.288 & $7.80 \mathrm{E}-14$ & 0.0001 & 0.78 & -0.189 & $2.40 \mathrm{E}-07$ & 0.0001 \\
\hline rs485425 & 11 & C & G & 0.45 & -0.229 & $5.30 \mathrm{E}-13$ & 0.0001 & 0.45 & -0.141 & $3.40 \mathrm{E}-06$ & 0.0001 \\
\hline rs4630328 & 11 & G & A & 0.62 & 0.237 & $6.60 \mathrm{E}-13$ & 0.0001 & 0.62 & 0.228 & 4.30E-13 & 0.0002 \\
\hline rs113443718 & 16 & G & $A$ & 0.69 & 0.251 & $9.80 \mathrm{E}-13$ & 0.0001 & 0.69 & 0.192 & $1.90 \mathrm{E}-08$ & 0.0001 \\
\hline rs378421 & 16 & G & $A$ & 0.58 & 0.228 & $1.40 \mathrm{E}-12$ & 0.0001 & 0.58 & 0.181 & $2.00 \mathrm{E}-08$ & 0.0001 \\
\hline rs838145 & 19 & G & $A$ & 0.46 & 0.229 & $2.60 \mathrm{E}-12$ & 0.0001 & 0.46 & 0.216 & 4.70E-12 & 0.0002 \\
\hline rs6969458 & 7 & G & $A$ & 0.53 & -0.227 & $5.00 \mathrm{E}-12$ & 0.0001 & 0.53 & -0.182 & $6.40 \mathrm{E}-09$ & 0.0001 \\
\hline rs75199129 & 2 & $A$ & $\mathrm{~T}$ & 0.95 & 0.510 & $1.00 \mathrm{E}-11$ & 0.0001 & 0.95 & 0.320 & 7.90E-06 & 0.0001 \\
\hline
\end{tabular}




\begin{tabular}{|c|c|c|c|c|c|c|c|c|c|c|c|}
\hline rs13413953 & 2 & $T$ & G & 0.64 & 0.228 & 1.60E-11 & 0.0001 & 0.64 & 0.168 & $1.80 \mathrm{E}-07$ & 0.0001 \\
\hline rs28929474 & 14 & C & $\mathrm{T}$ & 0.98 & 0.755 & 2.30E-11 & 0.0001 & 0.98 & 0.389 & $4.90 \mathrm{E}-04$ & 0.0001 \\
\hline rs4480324 & 1 & A & G & 0.30 & 0.239 & 2.60E-11 & 0.0001 & 0.29 & 0.145 & $3.80 \mathrm{E}-05$ & 0.0001 \\
\hline rs12124523 & 1 & C & $\mathrm{T}$ & 0.89 & 0.340 & 2.80E-11 & 0.0001 & 0.89 & 0.178 & $2.70 \mathrm{E}-04$ & 0.0001 \\
\hline rs74424378 & 9 & $T$ & G & 0.76 & 0.251 & 3.40E-11 & 0.0001 & 0.76 & 0.208 & $1.90 \mathrm{E}-08$ & 0.0001 \\
\hline rs77294902 & 17 & G & A & 0.78 & 0.252 & 5.00E-11 & 0.0001 & 0.78 & 0.161 & $1.50 \mathrm{E}-05$ & 0.0001 \\
\hline rs6452788 & 5 & G & A & 0.76 & -0.249 & $5.20 \mathrm{E}-11$ & 0.0001 & 0.76 & -0.223 & $8.20 \mathrm{E}-10$ & 0.0002 \\
\hline rs7786376 & 7 & A & G & 0.72 & -0.240 & $6.30 \mathrm{E}-11$ & 0.0001 & 0.72 & -0.193 & 4.90E-08 & 0.0001 \\
\hline rs7132908 & 12 & G & A & 0.62 & 0.206 & 1.50E-10 & 0.0001 & 0.62 & 0.119 & $1.80 \mathrm{E}-04$ & 0.0001 \\
\hline rs11860773 & 16 & $T$ & C & 0.80 & 0.264 & 1.60E-10 & 0.0001 & 0.80 & 0.204 & $1.40 \mathrm{E}-07$ & 0.0001 \\
\hline rs4815366 & 20 & G & T & 0.36 & -0.213 & 1.60E-10 & 0.0001 & 0.36 & -0.162 & $5.90 \mathrm{E}-07$ & 0.0001 \\
\hline rs2858088 & 4 & A & G & 0.38 & -0.208 & 3.50E-10 & 0.0001 & 0.38 & -0.129 & $5.00 \mathrm{E}-05$ & 0.0001 \\
\hline rs2959005 & 15 & C & $\mathrm{T}$ & 0.33 & -0.216 & 4.10E-10 & 0.0001 & 0.33 & -0.100 & $2.10 \mathrm{E}-03$ & 0.0000 \\
\hline rs9349379 & 6 & A & G & 0.59 & -0.207 & 4.30E-10 & 0.0001 & 0.59 & -0.146 & 4.30E-06 & 0.0001 \\
\hline rs748919 & 11 & $T$ & C & 0.79 & 0.245 & 4.60E-10 & 0.0001 & 0.79 & 0.131 & $4.90 \mathrm{E}-04$ & 0.0000 \\
\hline rs113441031 & 16 & C & T & 0.83 & 0.266 & 6.40E-10 & 0.0001 & 0.83 & 0.135 & $1.40 \mathrm{E}-03$ & 0.0000 \\
\hline rs109536 & 9 & G & C & 0.73 & -0.222 & $6.50 \mathrm{E}-10$ & 0.0001 & 0.73 & -0.102 & $2.70 \mathrm{E}-03$ & 0.0000 \\
\hline rs2717053 & 2 & G & C & 0.37 & -0.205 & $8.20 \mathrm{E}-10$ & 0.0001 & 0.37 & -0.074 & $2.10 \mathrm{E}-02$ & 0.0000 \\
\hline rs35572189 & 17 & G & A & 0.64 & -0.205 & $8.80 \mathrm{E}-10$ & 0.0001 & 0.64 & -0.089 & $5.40 \mathrm{E}-03$ & 0.0000 \\
\hline rs2274793 & 14 & C & T & 0.67 & 0.210 & $9.20 \mathrm{E}-10$ & 0.0001 & 0.67 & 0.154 & $1.70 \mathrm{E}-06$ & 0.0001 \\
\hline rs147711594 & 3 & G & $\mathrm{T}$ & 0.98 & 0.651 & $9.60 \mathrm{E}-10$ & 0.0001 & 0.98 & 0.416 & $2.90 \mathrm{E}-05$ & 0.0001 \\
\hline rs322764 & 7 & G & A & 0.44 & -0.197 & 1.00E-09 & 0.0001 & 0.44 & -0.131 & $3.70 \mathrm{E}-05$ & 0.0001 \\
\hline rs11692435 & 2 & G & A & 0.92 & -0.364 & 1.10E-09 & 0.0001 & 0.92 & -0.156 & $4.80 \mathrm{E}-03$ & 0.0000 \\
\hline rs7940127 & 11 & $T$ & C & 0.14 & -0.281 & 1.10E-09 & 0.0001 & 0.14 & -0.144 & $1.30 \mathrm{E}-03$ & 0.0000 \\
\hline rs11648570 & 16 & T & C & 0.89 & -0.318 & $1.30 \mathrm{E}-09$ & 0.0001 & 0.89 & -0.243 & $1.80 \mathrm{E}-06$ & 0.0001 \\
\hline
\end{tabular}




\begin{tabular}{|c|c|c|c|c|c|c|c|c|c|c|c|}
\hline rs72726477 & 4 & G & A & 0.88 & 0.304 & 1.30E-09 & 0.0001 & 0.88 & 0.136 & $4.40 \mathrm{E}-03$ & 0.0000 \\
\hline rs75543135 & 19 & $T$ & A & 0.88 & -0.309 & $1.70 \mathrm{E}-09$ & 0.0001 & 0.88 & -0.081 & $8.90 \mathrm{E}-02$ & 0.0000 \\
\hline rs56197131 & 7 & G & A & 0.80 & 0.239 & 2.70E-09 & 0.0001 & 0.80 & 0.170 & $1.20 \mathrm{E}-05$ & 0.0001 \\
\hline rs17177078 & 16 & C & $\mathrm{T}$ & 0.94 & 0.421 & 3.10E-09 & 0.0001 & 0.94 & 0.297 & $1.20 \mathrm{E}-05$ & 0.0001 \\
\hline rs324012 & 12 & C & $\mathrm{T}$ & 0.55 & -0.194 & 3.20E-09 & 0.0001 & 0.55 & -0.084 & $8.40 \mathrm{E}-03$ & 0.0000 \\
\hline rs142687608 & 16 & A & G & 0.98 & 0.661 & 7.50E-09 & 0.0001 & 0.98 & 0.508 & $5.30 \mathrm{E}-06$ & 0.0001 \\
\hline rs6136465 & 20 & G & A & 0.60 & 0.190 & 8.00E-09 & 0.0001 & 0.60 & 0.124 & $6.10 \mathrm{E}-05$ & 0.0001 \\
\hline rs7630012 & 3 & A & G & 0.57 & 0.186 & 8.00E-09 & 0.0001 & 0.57 & 0.165 & $2.00 \mathrm{E}-07$ & 0.0001 \\
\hline rs77123275 & 9 & C & $\mathrm{T}$ & 0.95 & -0.415 & $8.10 \mathrm{E}-09$ & 0.0001 & 0.95 & -0.284 & $5.70 \mathrm{E}-05$ & 0.0001 \\
\hline rs3809162 & 12 & A & G & 0.59 & -0.185 & 8.30E-09 & 0.0001 & 0.59 & -0.195 & $6.40 \mathrm{E}-10$ & 0.0002 \\
\hline rs28601761 & 8 & C & G & 0.58 & -0.193 & $8.40 \mathrm{E}-09$ & 0.0001 & 0.58 & -0.103 & $1.60 \mathrm{E}-03$ & 0.0000 \\
\hline rs9639559 & 7 & C & $\mathrm{T}$ & 0.28 & -0.213 & 8.40E-09 & 0.0001 & 0.27 & -0.136 & $9.70 \mathrm{E}-05$ & 0.0001 \\
\hline rs4775792 & 15 & $T$ & G & 0.37 & 0.192 & 8.50E-09 & 0.0001 & 0.37 & 0.094 & $4.50 \mathrm{E}-03$ & 0.0000 \\
\hline rs17446532 & 9 & C & $\mathrm{T}$ & 0.51 & -0.182 & $8.80 \mathrm{E}-09$ & 0.0001 & 0.51 & -0.106 & $8.20 \mathrm{E}-04$ & 0.0000 \\
\hline rs12899560 & 15 & C & $\mathrm{T}$ & 0.59 & -0.195 & $9.60 \mathrm{E}-09$ & 0.0001 & 0.59 & -0.096 & $2.20 \mathrm{E}-03$ & 0.0000 \\
\hline rs11773627 & 7 & T & c & 0.81 & -0.238 & $9.70 \mathrm{E}-09$ & 0.0001 & 0.81 & -0.093 & $2.10 \mathrm{E}-02$ & 0.0000 \\
\hline rs2117760 & 3 & C & A & 0.71 & 0.198 & 1.00E-08 & 0.0001 & 0.71 & 0.180 & $1.40 \mathrm{E}-07$ & 0.0001 \\
\hline rs7673993 & 4 & A & G & 0.58 & -0.187 & 1.10E-08 & 0.0001 & 0.58 & -0.161 & $3.60 \mathrm{E}-07$ & 0.0001 \\
\hline rs142488468 & 10 & G & c & 0.82 & 0.238 & $1.30 \mathrm{E}-08$ & 0.0001 & 0.82 & 0.146 & $1.90 \mathrm{E}-04$ & 0.0001 \\
\hline rs7499750 & 16 & A & C & 0.22 & 0.217 & 1.40E-08 & 0.0001 & 0.23 & 0.170 & $6.00 \mathrm{E}-06$ & 0.0001 \\
\hline rs2584448 & 4 & $T$ & G & 0.43 & -0.187 & $1.70 \mathrm{E}-08$ & 0.0001 & 0.43 & -0.159 & $5.00 \mathrm{E}-07$ & 0.0001 \\
\hline rs17884691 & 22 & G & A & 0.75 & 0.208 & 2.00E-08 & 0.0001 & 0.75 & 0.155 & $2.60 \mathrm{E}-05$ & 0.0001 \\
\hline rs756747 & 7 & $T$ & G & 0.48 & -0.183 & 2.00E-08 & 0.0001 & 0.48 & -0.111 & $2.30 \mathrm{E}-04$ & 0.0001 \\
\hline rs11030084 & 11 & C & $\mathrm{T}$ & 0.81 & 0.228 & 2.20E-08 & 0.0001 & 0.81 & 0.113 & $3.30 \mathrm{E}-03$ & 0.0000 \\
\hline rs1713675 & 11 & A & G & 0.51 & -0.183 & 2.40E-08 & 0.0001 & 0.51 & -0.132 & 4.40E-05 & 0.0001 \\
\hline
\end{tabular}




\begin{tabular}{|c|c|c|c|c|c|c|c|c|c|c|c|}
\hline rs2756185 & 6 & G & A & 0.93 & 0.337 & 2.60E-08 & 0.0001 & 0.93 & 0.066 & 2.10E-01 & 0.0000 \\
\hline rs11079849 & 17 & C & $\mathrm{T}$ & 0.67 & -0.195 & $2.80 \mathrm{E}-08$ & 0.0001 & 0.67 & -0.111 & $9.20 \mathrm{E}-04$ & 0.0000 \\
\hline rs118784 & 5 & $\mathrm{~T}$ & A & 0.50 & -0.178 & $3.20 \mathrm{E}-08$ & 0.0001 & 0.50 & -0.110 & 3.30E-04 & 0.0001 \\
\hline rs78621285 & 16 & A & $\mathrm{T}$ & 0.91 & 0.312 & $3.20 \mathrm{E}-08$ & 0.0001 & 0.91 & 0.175 & $1.10 \mathrm{E}-03$ & 0.0000 \\
\hline rs1788820 & 18 & $A$ & G & 0.35 & -0.186 & $3.40 \mathrm{E}-08$ & 0.0001 & 0.34 & -0.156 & $1.70 \mathrm{E}-06$ & 0.0001 \\
\hline rs2068650 & 5 & A & C & 0.53 & 0.178 & 3.70E-08 & 0.0001 & 0.53 & 0.145 & $2.60 \mathrm{E}-06$ & 0.0001 \\
\hline rs6690101 & 1 & $\mathrm{~T}$ & C & 0.46 & -0.178 & $3.90 \mathrm{E}-08$ & 0.0001 & 0.46 & -0.114 & $2.80 \mathrm{E}-04$ & 0.0001 \\
\hline rs1788030 & 18 & C & $\mathrm{T}$ & 0.54 & 0.179 & 4.70E-08 & 0.0001 & 0.54 & 0.096 & 3.00E-03 & 0.0000 \\
\hline rs11090045 & 22 & G & A & 0.69 & 0.194 & 4.80E-08 & 0.0001 & 0.69 & 0.129 & $1.50 \mathrm{E}-04$ & 0.0001 \\
\hline Combined & & & & & & & 0.0094 & & & & 0.0095 \\
\hline
\end{tabular}

Supplementary Table 4. Power calculation for MR analysis

\begin{tabular}{|l|r|r|r|r|r|}
\hline Outcome & Number of Controls & Number of cases & OR>1.15 & OR>1.20 & OR>1.30 \\
\hline Breast cancer & 105974 & 122977 & 0.91 & 0.99 & 0.99 \\
\hline Ovarian cancer & 40941 & 22406 & 0.4 & 0.62 & 0.91 \\
\hline
\end{tabular}

Power for MR analysis estimated for a 1SD change in alcohol intake ( 1.4 stand drinks/day) using $\mathrm{mRnd}$ power calculator (https://shiny.cnsgenomics.com/mRnd/). Variance explained by instrument is set to be $r^{2}=0.95 \%$. 
Supplementary Table 5. Estimated hazard ratios for the association between daily alcohol consumption and breast and ovarian cancer in the CCHS+CGPS for different consumption levels.

\begin{tabular}{|c|c|c|c|}
\hline Breast cancer & Total sample size & HR (adjusted model) & P-value \\
\hline \multicolumn{4}{|c|}{ Daily number of drinks ( 1 drink $=12 \mathrm{~g}$ ) } \\
\hline $0.0-0.9$ & 44451 & $0.91(0.82,1.00)$ & 0.05 \\
\hline $1.0-1.9$ & 21435 & REF & - \\
\hline 2.0-2.9 & 8156 & $1.11(0.97,1.27)$ & 0.13 \\
\hline 3.0-3.0 & 2765 & $1.24(1.02,1.51)$ & 0.03 \\
\hline 4.0-4.9 & 871 & $1.38(1.00,1.90)$ & 0.05 \\
\hline $5.0-9.9$ & 550 & $1.36(0.91,2.03)$ & 0.13 \\
\hline \multirow[t]{2}{*}{$>=10$} & 38 & $4.3(1.61,11.49)$ & 0.004 \\
\hline & & & P-trend $=0.02$ \\
\hline \multicolumn{4}{|l|}{ Ovarian cancer } \\
\hline \multicolumn{4}{|c|}{ Daily number of drinks ( 1 drink=12g) } \\
\hline $0.0-0.9$ & 45601 & $1.19(0.91,1.55)$ & 0.22 \\
\hline $1.0-1.9$ & 22242 & REF & \\
\hline 2.0-2.9 & 8510 & $1.19(0.81,1.74)$ & 0.37 \\
\hline 3.0-3.0 & 2873 & $0.62(0.29,1.35)$ & 0.23 \\
\hline 4.0-4.9 & 916 & $2.89(1.50,5.58)$ & 0.002 \\
\hline $5.0-9.9$ & 566 & $1.87(0.69,5.12)$ & 0.22 \\
\hline \multirow[t]{2}{*}{$>=10$} & no events & & \\
\hline & & & P-trend $=0.32$ \\
\hline
\end{tabular}

Analyses used age as the underlying time, and were adjusted for cohort (CCHS or CGPS), birth year, and examination year. For both cancers, final consumption category $(>=10)$ was omitted from trend test. 
Supplementary Table 6. Estimated hazard ratio for the association between daily alcohol drinks with breast cancer in the KARMA study for different consumption levels.

\begin{tabular}{|l|l|l|l|}
\hline Study & $\begin{array}{l}\text { Category } \\
\text { (daily alcohol intake, in grams) }\end{array}$ & HR (only age adjusted) & HR (full adjusted model) \\
\hline KARMA & $0.1-9.9 \mathrm{~g}$ & $1.13(0.93,1.36)$ & $1.10(0.91,1.34)$ \\
\hline KARMA & $10-20 \mathrm{~g}$ & $1.18(0.79,1.76)$ & $1.14(0.76,1.69)$ \\
\hline KARMA & $20-30 \mathrm{~g}$ & $1.30(0.62,2.74)$ & $1.25(0.60,2.64)$ \\
\hline
\end{tabular}

Supplementary Table 7. Estimated hazard ratio for the association between daily alcohol drinks with breast cancer in the KARMA study stratified by ER status of breast cancer.

\begin{tabular}{|l|l|l|l|l|}
\hline Study & ER Status & Events & HR (only age adjusted) & HR (full adjusted model) \\
\hline KARMA & All breast cancer & 985 & $1.09(0.99,1.20)$ & $1.07(0.97,1.19)$ \\
\hline KARMA & ER+ breast cancer & 867 & $1.10(0.99,1.23)$ & $1.09(0.98,1.21)$ \\
\hline KARMA & ER- breast cancer & 118 & $0.97(0.73,1.30)$ & $0.99(0.74,1.34)$ \\
\hline
\end{tabular}


Supplementary Table 8. Comparison of age- and fully-adjusted hazard ratios for the association between daily alcohol intake and breast and ovarian cancer risk for each study. Age was used as the underlying time variable. Participants were censored at the last visit, death or event, whichever came first.

\begin{tabular}{|c|c|c|c|c|c|c|}
\hline \multirow[b]{2}{*}{ Study } & \multicolumn{3}{|c|}{ Only age adjustment } & \multicolumn{3}{|c|}{ Multifactorial adjustment } \\
\hline & Events & Participants & HR_age adjusted & Events & Participants & HR_fully adjusted \\
\hline \multicolumn{7}{|l|}{ Breast } \\
\hline KARMA & 985 & 59918 & $1.12(1.01,1.24)$ & 985 & 59918 & $1.07(0.97,1.19)$ \\
\hline $\mathrm{CCHS}+\mathrm{CGPS}$ & 2312 & 65803 & $1.07(1.04,1.10)$ & 2055 & 63560 & $1.09(1.05,1.13)$ \\
\hline UK Biobank & 4081 & 141008 & $1.08(1.06,1.10)$ & 1787 & 64622 & $1.04(1.01,1.07)$ \\
\hline \multicolumn{7}{|l|}{ Ovarian } \\
\hline $\mathrm{CCHS}+\mathrm{CGPS}$ & 327 & 67981 & $1.05(0.97,1.14)$ & 287 & 62867 & $1.07(0.99,1.16)$ \\
\hline UK Biobank & 427 & 137394 & $0.97(0.90,1.04)$ & 187 & 61267 & $0.92(0.83,1.03)$ \\
\hline
\end{tabular}

The crude HR model is adjusted for recruitment age, top 10 ancestral principal components (UKB only) and Townsend deprivation index (UKB only). The adjusted model incorporates the following additional covariates: coffee intake, BMI, height, smoking pack years, menopausal status, number of live births, ever smoked, education, duration of moderate and vigorous physical activities and age at menarche. Given that a sizeable proportion of the UK Biobank participants are cryptically related, estimates obtained from the UK Biobank had been adjusted for cryptic relatedness (i.e. related individuals removed within and between cancer cases and healthy individuals undiagnosed with any cancer). 
Supplementary Table 9. Comparison of pooled HR estimates obtained through missing covariate data imputation against original estimates in the UK Biobank breast and ovarian cancer cohort.

\begin{tabular}{|c|c|c|c|c|}
\hline Outcome & Events & Participants & HR (95\% Cl) & P-value \\
\hline \multicolumn{5}{|c|}{ Age-adjusted HR model } \\
\hline Breast cancer & 4081 & 141008 & $1.08(1.06,1.10)$ & $3.20 \mathrm{E}-15$ \\
\hline Ovarian cancer & 427 & 137394 & $0.97(0.90,1.04)$ & 0.38 \\
\hline \multicolumn{5}{|c|}{ Multivariable-adjusted HR model } \\
\hline Breast cancer & 1787 & 64622 & $1.04(1.01,1.07)$ & 0.01 \\
\hline Ovarian cancer & 187 & 61267 & $0.92(0.83,1.03)$ & 0.16 \\
\hline \multicolumn{5}{|c|}{ Pooled multivariable-adjusted HR model using imputed covariate data } \\
\hline Breast cancer & 4081 & 141008 & $1.02(1.00,1.03)$ & $8.5 \mathrm{E}-3$ \\
\hline Ovarian cancer & 427 & 137394 & $0.94(0.90,0.99)$ & 0.01 \\
\hline
\end{tabular}

The crude HR model is adjusted for recruitment age, top 10 ancestral principal components (UKB only) and Townsend deprivation index (UKB only). The adjusted model incorporates the following additional covariates: coffee intake, BMI, height, smoking pack years, menopausal status, number of live births, ever smoked, education, duration of moderate and vigorous physical activities and age at menarche. The pooled HR estimates were averaged across 5 imputed datasets. Note that the cancer outcome of interest, recruitment age, and diagnosis age were not imputed. 
Supplementary Table 10. Global test for heterogeneity on MR causal estimates. SNP-outliers are detected using the conventional Cochran $\mathrm{Q}$-statistics (with $\mathrm{df}=1$ ), where SNPs that have a heterogeneity score $>3.84$ are filtered. The causal estimate derived from the filtered set of SNPs are reported in the main analysis.

\begin{tabular}{|c|c|c|c|c|c|c|c|}
\hline \multirow[b]{2}{*}{ Trait } & \multirow[b]{2}{*}{ Methods } & \multicolumn{3}{|c|}{$\begin{array}{l}\text { Before filtering heterogenous SNP- } \\
\text { effects }\end{array}$} & \multicolumn{3}{|c|}{ After filtering heterogenous SNP-effects } \\
\hline & & nsnps & $\begin{array}{l}\text { cochran Q- } \\
\text { stats }\end{array}$ & Q-stat pvalue & nsnps & $\begin{array}{l}\text { cochran Q- } \\
\text { stats }\end{array}$ & $\begin{array}{l}\text { Q-stat } \\
\text { pvalue }\end{array}$ \\
\hline \multirow[t]{2}{*}{ All BrCa } & IVW(main) & 34 & 82.4 & 2.53E-06 & 29 & 26.6 & 0.54 \\
\hline & MR-Egger & 34 & 81.9 & $1.80 \mathrm{E}-06$ & 29 & 26.6 & 0.49 \\
\hline \multirow[t]{2}{*}{ All EOC } & IVW(main) & 34 & 73.7 & 6.19E-05 & 33 & 24.3 & 0.83 \\
\hline & MR-Egger & 34 & 73.3 & 4.45E-05 & 33 & 22.8 & 0.86 \\
\hline & & & & & & & \\
\hline
\end{tabular}

The variant(s) that was dropped after heterogeneity filtering were rs11648570, rs2117760, rs61873510 and rs62055546 for the breast cancer MR analysis. The variant that was dropped was rs 62055546 for the ovarian cancer MR analysis. 
Supplementary Table 11. Comparison of MR estimates across alternative MR methods before and after filtering for SNP-heterogeneity. For MR-Egger(bootstrap), 1000 bootstrap iterations were performed to obtain reliable standard errors for the causal estimates. PWM stands for the penalized weighted median model.

\begin{tabular}{|c|c|c|c|c|c|c|c|c|c|}
\hline \multirow[t]{2}{*}{ Outcome } & \multirow[t]{2}{*}{ MR-model } & \multicolumn{4}{|c|}{$\begin{array}{l}\text { Before filtering for SNP- } \\
\text { heterogeneity }\end{array}$} & \multicolumn{4}{|c|}{$\begin{array}{l}\text { After filtering for SNP- } \\
\text { heterogeneity }\end{array}$} \\
\hline & & P-value & OR & L_95Cl & U_95Cl & $\begin{array}{l}\mathrm{P} \text { - } \\
\text { value }\end{array}$ & OR & L_95Cl & U_95C \\
\hline \multirow{4}{*}{$\begin{array}{l}\text { All Breast } \\
\text { cancers }\end{array}$} & MR-Egger & 0.96 & 1.00 & 0.86 & 1.17 & 0.83 & 0.99 & 0.90 & 1.09 \\
\hline & PWM & 0.50 & 0.97 & 0.89 & 1.06 & 0.51 & 0.97 & 0.89 & 1.06 \\
\hline & $\begin{array}{l}\text { IVW (random } \\
\text { effect) }\end{array}$ & 0.53 & 1.03 & 0.93 & 1.14 & 0.78 & 0.99 & 0.93 & 1.06 \\
\hline & $\begin{array}{l}\text { IVW (fixed } \\
\text { effect) }\end{array}$ & 0.31 & 1.03 & 0.97 & 1.10 & 0.78 & 0.99 & 0.93 & 1.06 \\
\hline \multirow[t]{4}{*}{ All EOC } & MR-Egger & 0.27 & 0.84 & 0.62 & 1.14 & 0.17 & 0.86 & 0.71 & 1.06 \\
\hline & PWM & 0.07 & 0.84 & 0.70 & 1.01 & 0.003 & 0.73 & 0.59 & 0.90 \\
\hline & $\begin{array}{l}\text { IVW (random } \\
\text { effect) }\end{array}$ & 0.23 & 0.89 & 0.73 & 1.08 & 0.39 & 0.95 & 0.85 & 1.07 \\
\hline & $\begin{array}{l}\text { IVW (fixed } \\
\text { effect) }\end{array}$ & 0.07 & 0.89 & 0.78 & 1.01 & 0.46 & 0.95 & 0.83 & 1.08 \\
\hline
\end{tabular}


Supplementary Table 12. Multivariable MR analysis adjusting for the effect on adiposity, smoking behaviour and education attainment. Changes in the MVMR estimate before and after filtering out SNPs with heterogeneous effect sizes indicate that the unadjusted estimates were largely driven by outliers. Estimated OR reflect the change in risk per one SD increase in the risk factors.

\begin{tabular}{|c|c|c|c|c|c|c|c|c|c|c|c|}
\hline \multirow[b]{2}{*}{ Risk factors } & \multirow[b]{2}{*}{ Outcome } & \multicolumn{5}{|c|}{$\begin{array}{l}\text { Before filtering for heterogenous } \\
\text { variants }\end{array}$} & \multicolumn{5}{|c|}{$\begin{array}{l}\text { After filtering for heterogenous } \\
\text { variants }\end{array}$} \\
\hline & & nsnp & pval & OR & Cl_low & Cl_upper & nsnp & pval & OR & CI_low & Cl_upper \\
\hline Cigarette/day & $\begin{array}{l}\text { Breast } \\
\text { cancer }\end{array}$ & 34 & 0.02 & 1.03 & 1.01 & 1.06 & 30 & 0.00 & 1.03 & 1.01 & 1.05 \\
\hline $\begin{array}{l}\text { Education } \\
\text { attainment }\end{array}$ & $\begin{array}{l}\text { Breast } \\
\text { cancer }\end{array}$ & 34 & 0.08 & 1.67 & 0.95 & 2.96 & 30 & 0.20 & 1.34 & 0.86 & 2.09 \\
\hline $\begin{array}{l}\text { estimated } \\
\text { standard } \\
\text { drinks/week }\end{array}$ & $\begin{array}{l}\text { Breast } \\
\text { cancer }\end{array}$ & 34 & 0.02 & 1.10 & 1.02 & 1.19 & 30 & 0.31 & 1.03 & 0.97 & 1.10 \\
\hline $\begin{array}{l}\text { Waist-Hip Ratio } \\
\text { adjusted for BMI }\end{array}$ & $\begin{array}{l}\text { Breast } \\
\text { cancer }\end{array}$ & 34 & 0.00 & 0.45 & 0.29 & 0.70 & 30 & 0.03 & 0.69 & 0.49 & 0.97 \\
\hline BMI & $\begin{array}{l}\text { Breast } \\
\text { cancer }\end{array}$ & 34 & 0.92 & 1.00 & 0.93 & 1.07 & 30 & 0.95 & 1.00 & 0.95 & 1.06 \\
\hline Cigarette/day & $\begin{array}{l}\text { Ovarian } \\
\text { cancer }\end{array}$ & 34 & 0.19 & 1.04 & 0.98 & 1.11 & 33 & 0.01 & 1.05 & 1.01 & 1.09 \\
\hline $\begin{array}{l}\text { Education } \\
\text { attainment }\end{array}$ & $\begin{array}{l}\text { Ovarian } \\
\text { cancer }\end{array}$ & 34 & 0.04 & 0.26 & 0.07 & 0.94 & 33 & 0.50 & 0.76 & 0.34 & 1.69 \\
\hline $\begin{array}{l}\text { estimated } \\
\text { standard } \\
\text { drinks/week }\end{array}$ & $\begin{array}{l}\text { Ovarian } \\
\text { cancer }\end{array}$ & 34 & 0.03 & 0.82 & 0.95 & 1.00 & 33 & 0.65 & 0.97 & 0.87 & 1.09 \\
\hline $\begin{array}{l}\text { Waist-Hip Ratio } \\
\text { adjusted for BMI }\end{array}$ & $\begin{array}{l}\text { Ovarian } \\
\text { cancer }\end{array}$ & 34 & 0.42 & 1.53 & 0.55 & 4.26 & 33 & 0.11 & 0.59 & 0.31 & 1.13 \\
\hline BMI & $\begin{array}{l}\text { Ovarian } \\
\text { cancer }\end{array}$ & 4 & 0.43 & 0.94 & 0.79 & 1.11 & 33 & 0.98 & 1.00 & 0.91 & 1.11 \\
\hline
\end{tabular}

The variant(s) that was dropped after heterogeneity filtering were rs11648570, rs2117760, rs61873510 and rs62055546 for the breast cancer MR analysis. The variant that was dropped was rs 62055546 for the ovarian cancer MR analysis. PheWAS analysis reveal that the dropped variant rs62055546 in both the breast and ovarian cancer analyses was strongly associated with changes in red blood cell count (http://geneatlas.roslin.ed.ac.uk/phewas/?variant=rs62055546\&representation=table). 
Supplementary Table 13. Comparison of instrument strength across various sets of instrument from UK Biobank drinks/week GWAS. The median value (and the interquartile range) for the partial Fstatistics for each set of instruments were reported.

\begin{tabular}{|l|r|r|r|r|}
\hline $\begin{array}{l}\text { Test } \\
\text { statistics }\end{array}$ & $\begin{array}{l}\text { Alcohol intake } \\
\text { (combined sex) }\end{array}$ & $\begin{array}{l}\text { Alcohol intake (combined } \\
\text { sex) }\end{array}$ & Alcohol intake (females only) \\
\hline $\begin{array}{l}\text { Number of } \\
\text { SNPs }\end{array}$ & 77 & 34 & 34 \\
\hline Total r2 & 0.009 & 0.006 & 0.007 \\
\hline $\begin{array}{l}\text { Partial } \\
\text { F-stat }\end{array}$ & $30.31[26.6-37.0]$ & $37.3[29.3-46.3]$ & $22.2[19.5-30.3]$ \\
\hline total F & 50 & 75 & 41.7 \\
\hline & Variants identified from & $\begin{array}{l}\text { Variants identified from combined sex } \\
\text { GWAS that replicate in female only } \\
\text { GWAS (p<1e-5), using EAF and effect }\end{array}$ \\
\hline Description & $\begin{array}{l}\text { Variants identified } \\
\text { from combined sex } \\
\text { alcohol GWAS }\end{array}$ & $\begin{array}{l}\text { combined sex GWAS that } \\
\text { replicate in female only } \\
\text { GWAS (p<1e-5) }\end{array}$ & $\begin{array}{l}\text { GWes from female only GWAS } \\
\text { sizem }\end{array}$ \\
\hline
\end{tabular}


Supplementary Table 14. Evidence of replication of UKBB estimated drinks/week (females only) SNPassociation in the GSCAN drinks/week GWAS summary statistics

\begin{tabular}{|c|c|c|c|c|c|c|c|c|c|}
\hline SNP & $\mathrm{CHR}$ & NEA & EA & EAF & $\begin{array}{l}\text { BETA } \\
\text { GSCAN }\end{array}$ & SE_GSCAN & N_GSCAN & $\begin{array}{l}\text { PVALUE_- } \\
\text { GSCAN }\end{array}$ & PVAL_UKBB \\
\hline rs1260326 & 2 & $T$ & C & 0.60 & 0.024 & 0.002 & 532340 & 3.33E-33 & 4.60E-23 \\
\hline rs75199129 & 2 & A & $\mathrm{T}$ & 0.04 & -0.026 & 0.005 & 513023 & 8.44E-09 & 7.90E-06 \\
\hline rs 1004787 & 2 & $G$ & A & 0.58 & 0.015 & 0.002 & 526940 & $3.31 \mathrm{E}-15$ & $1.10 \mathrm{E}-13$ \\
\hline rs 13413953 & 2 & $T$ & G & 0.34 & -0.012 & 0.002 & 529000 & 5.35E-09 & $1.80 \mathrm{E}-07$ \\
\hline rs7630012 & 3 & $A$ & $\mathrm{G}$ & 0.46 & -0.007 & 0.002 & 535602 & 0.000423 & 2.00E-07 \\
\hline rs2117760 & 3 & $C$ & A & 0.28 & -0.009 & 0.002 & 524866 & $1.01 \mathrm{E}-05$ & $1.40 \mathrm{E}-07$ \\
\hline rs9822731 & 3 & $\mathrm{~T}$ & C & 0.22 & 0.017 & 0.002 & 531166 & 5.03E-14 & $2.40 \mathrm{E}-07$ \\
\hline rs11940694 & 4 & A & G & 0.60 & 0.028 & 0.002 & 527865 & $3.11 \mathrm{E}-46$ & $1.40 \mathrm{E}-26$ \\
\hline rs7673993 & 4 & A & G & 0.41 & 0.012 & 0.002 & 529073 & 2.65E-09 & 3.60E-07 \\
\hline rs1229984 & 4 & $\mathrm{~T}$ & C & 0.95 & 0.188 & 0.006 & 514602 & $1.60 \mathrm{E}-203$ & $6.80 \mathrm{E}-121$ \\
\hline rs 1302808 & 4 & C & A & 0.19 & 0.024 & 0.002 & 516605 & $1.36 \mathrm{E}-23$ & $6.50 \mathrm{E}-16$ \\
\hline rs2584448 & 4 & $\mathrm{~T}$ & G & 0.54 & 0.010 & 0.002 & 531331 & 1.83E-07 & $5.00 \mathrm{E}-07$ \\
\hline rs13107325 & 4 & C & $\mathrm{T}$ & 0.07 & -0.036 & 0.004 & 528164 & 1.23E-20 & $6.70 \mathrm{E}-07$ \\
\hline rs6452788 & 5 & $G$ & A & 0.27 & 0.012 & 0.002 & 535356 & $3.58 \mathrm{E}-07$ & $8.20 \mathrm{E}-10$ \\
\hline rs2068650 & 5 & A & C & 0.47 & -0.009 & 0.002 & 527780 & 2.38E-06 & $2.60 \mathrm{E}-06$ \\
\hline rs9349379 & 6 & A & G & 0.40 & 0.009 & 0.002 & 526515 & 4.90E-06 & 4.30E-06 \\
\hline rs7786376 & 7 & A & G & 0.27 & 0.011 & 0.002 & 525890 & 1.01E-06 & $4.90 \mathrm{E}-08$ \\
\hline rs6969458 & 7 & $G$ & A & 0.46 & 0.013 & 0.002 & 509646 & $5.20 \mathrm{E}-11$ & $6.40 \mathrm{E}-09$ \\
\hline rs74424378 & 9 & $\mathrm{~T}$ & G & 0.26 & -0.011 & 0.002 & 530826 & $1.72 \mathrm{E}-06$ & $1.90 \mathrm{E}-08$ \\
\hline rs61873510 & 10 & $G$ & $\mathrm{~T}$ & 0.31 & -0.011 & 0.002 & 500397 & 8.83E-08 & $5.80 \mathrm{E}-08$ \\
\hline rs11604680 & 11 & A & G & 0.32 & -0.015 & 0.002 & 526748 & $2.87 E-12$ & 5.80E-07 \\
\hline rs4630328 & 11 & G & A & 0.35 & -0.014 & 0.002 & 531293 & $3.80 \mathrm{E}-12$ & $4.30 \mathrm{E}-13$ \\
\hline rs485425 & 11 & C & G & 0.54 & 0.010 & 0.002 & 532602 & 2.13E-07 & $3.40 \mathrm{E}-06$ \\
\hline rs3809162 & 12 & A & G & 0.41 & 0.010 & 0.002 & 527315 & 4.61E-07 & $6.40 \mathrm{E}-10$ \\
\hline rs2274793 & 14 & C & $\mathrm{T}$ & 0.33 & -0.012 & 0.002 & 531843 & $3.15 \mathrm{E}-09$ & $1.70 \mathrm{E}-06$ \\
\hline rs7499750 & 16 & A & C & 0.78 & -0.009 & 0.002 & 534136 & 0.000192 & $6.00 \mathrm{E}-06$ \\
\hline rs378421 & 16 & $G$ & A & 0.41 & -0.013 & 0.002 & 508328 & $2.26 \mathrm{E}-11$ & $2.00 \mathrm{E}-08$ \\
\hline rs 113443718 & 16 & $G$ & A & 0.28 & -0.011 & 0.002 & 510879 & $5.26 \mathrm{E}-08$ & $1.90 \mathrm{E}-08$ \\
\hline rs142687608 & 16 & $A$ & $G$ & 0.02 & -0.021 & 0.007 & 489914 & 0.00226 & 5.30E-06 \\
\hline
\end{tabular}




\begin{tabular}{|l|r|l|l|r|r|r|r|r|r|}
\hline rs11648570 & 16 & T & C & 0.11 & 0.019 & 0.003 & 526153 & $3.43 E-09$ & $1.80 \mathrm{E}-06$ \\
\hline rs11860773 & 16 & T & C & 0.18 & -0.015 & 0.002 & 515422 & $8.35 \mathrm{E}-10$ & $1.40 \mathrm{E}-07$ \\
\hline rs62055546 & 17 & A & C & 0.20 & -0.020 & 0.002 & 529432 & $3.06 \mathrm{E}-17$ & $1.10 \mathrm{E}-11$ \\
\hline rs1788820 & 18 & A & G & 0.65 & 0.007 & 0.002 & 535226 & 0.000374 & $1.70 \mathrm{E}-06$ \\
\hline rs838145 & 19 & G & A & 0.58 & -0.016 & 0.002 & 521587 & $3.87 \mathrm{E}-16$ & $4.70 \mathrm{E}-12$ \\
\hline rs4815366 & 20 & G & T & 0.67 & 0.009 & 0.002 & 534788 & $2.70 \mathrm{E}-06$ & $5.90 \mathrm{E}-07$ \\
\hline
\end{tabular}

Supplementary Table 15. MR Steiger Z-test for directionality.

\begin{tabular}{|l|l|r|r|l|l|}
\hline Outcome & Exposure & $\begin{array}{l}\boldsymbol{r}^{2} \text { on } \\
\text { exposure }\end{array}$ & $\begin{array}{l}\boldsymbol{r}^{2} \text { on } \\
\text { outcome }\end{array}$ & $\begin{array}{l}\text { Correct causal } \\
\text { direction }\end{array}$ & $\begin{array}{l}\text { Directionality test p- } \\
\text { value }\end{array}$ \\
\hline $\begin{array}{l}\text { Breast } \\
\text { cancer }\end{array}$ & $\begin{array}{l}\text { alcohol } \\
\text { (drinks/week) }\end{array}$ & 0.007 & 0.00024 & TRUE & $<1 \mathrm{e}-300$ \\
\hline $\begin{array}{l}\text { Ovarian } \\
\text { cancer }\end{array}$ & $\begin{array}{l}\text { alcohol } \\
\text { (drinks/week) }\end{array}$ & 0.007 & 0.00078 & TRUE & $<1 \mathrm{e}-300$ \\
\hline
\end{tabular}

Supplementary Table 16. Estimate of the MR-Egger intercept for the MR analysis between alcohol intake with breast and ovarian cancer risk. For both cancer outcomes, the MR Egger intercept was not significantly different from zero, presenting limited evidence against the presence of directional pleiotropy biasing the IVW results.

\begin{tabular}{|l|r|r|r|r|r|}
\hline Outcome & Intercept & se(intercept) & Pvalue & \multicolumn{1}{l|}{$\begin{array}{l}\text { lower 95\% } \\
\text { C.I. }\end{array}$} & upper 95\% C.I. \\
\hline All Breast cancer & 0.002 & 0.003 & 0.483 & -0.004 & 0.008 \\
\hline & & & & & \\
\hline All EOC & -0.001 & 0.004 & 0.752 & -0.009 & 0.006 \\
\hline
\end{tabular}




\section{Supplementary Table 17. Pleiotropy assessment on ADH1B SNP rs1229984 using the online}

Phenoscanner database. Beta refer to the magnitude of association on the traits per effect allele (T), the none effect allele for the SNP is C. Note that traits that are directly related to alcohol drinking (e.g. alcohol dependence) are excluded. Traits with $\mathrm{N}$ _cases being zero are quantitative traits. Only association estimates derived from Europeans were included.

\begin{tabular}{|c|c|c|c|c|c|c|c|}
\hline Trait & Beta & se & P-value & direction & $\mathbf{N}$ & N_cases & N_controls \\
\hline $\begin{array}{l}\text { Upper } \\
\text { aerodigestive } \\
\text { tract cancers }\end{array}$ & 0.4447 & 0.0476 & $1 \mathrm{E}-20$ & + & - & - & - \\
\hline $\begin{array}{l}\text { Self-reported } \\
\text { gout }\end{array}$ & 0.0084 & 0.001 & 3.6E-18 & + & 337159 & 4807 & 332352 \\
\hline $\begin{array}{l}\text { Reason for } \\
\text { reducing } \\
\text { amount of } \\
\text { alcohol drunk: } \\
\text { health } \\
\text { precaution }\end{array}$ & -0.051 & 0.0063 & $8 \mathrm{E}-16$ & - & 124798 & 40728 & 84070 \\
\hline $\begin{array}{l}\text { Treatment } \\
\text { with } \\
\text { allopurinol }\end{array}$ & 0.0068 & 0.0009 & 6.1E-15 & + & 337159 & 3819 & 333340 \\
\hline $\begin{array}{l}\text { Leg fat mass } \\
\text { right }\end{array}$ & -0.042 & 0.0066 & $9.4 \mathrm{E}-11$ & - & 331293 & 0 & 331293 \\
\hline $\begin{array}{l}\text { Systolic blood } \\
\text { pressure }\end{array}$ & -0.052 & 0.0083 & 3.3E-10 & - & 317754 & 0 & 317754 \\
\hline $\begin{array}{l}\text { Leg fat mass } \\
\text { left }\end{array}$ & -0.04 & 0.0065 & $9.5 \mathrm{E}-10$ & - & 331275 & 0 & 331275 \\
\hline $\begin{array}{l}\text { Leg fat } \\
\text { percentage } \\
\text { right }\end{array}$ & -0.03 & 0.0052 & 6.5E-09 & - & 331296 & 0 & 331296 \\
\hline $\begin{array}{l}\text { Whole body } \\
\text { fat mass }\end{array}$ & -0.045 & 0.008 & 1.4E-08 & - & 330762 & 0 & 330762 \\
\hline $\begin{array}{l}\text { Leg fat } \\
\text { percentage } \\
\text { left }\end{array}$ & -0.028 & 0.0051 & 5E-08 & - & 331278 & 0 & 331278 \\
\hline
\end{tabular}




\begin{tabular}{|c|c|c|c|c|c|c|c|}
\hline $\begin{array}{l}\text { Body mass } \\
\text { index }\end{array}$ & -0.044 & 0.0081 & $6.5 \mathrm{E}-08$ & - & 336107 & 0 & 336107 \\
\hline $\begin{array}{l}\text { Vascular or } \\
\text { heart } \\
\text { problems } \\
\text { diagnosed by } \\
\text { doctor: none } \\
\text { of the above }\end{array}$ & 0.02 & 0.0037 & 8.9E-08 & + & 336683 & 236530 & 100153 \\
\hline $\begin{array}{l}\text { Vascular or } \\
\text { heart } \\
\text { problems } \\
\text { diagnosed by } \\
\text { doctor: high } \\
\text { blood pressure }\end{array}$ & -0.019 & 0.0036 & 1.1E-07 & - & 336683 & 91033 & 245650 \\
\hline $\begin{array}{l}\text { Sodium in } \\
\text { urine }\end{array}$ & 0.0422 & 0.008 & $1.2 \mathrm{E}-07$ & + & 326831 & 0 & 326831 \\
\hline $\begin{array}{l}\text { Arm fat mass } \\
\text { left }\end{array}$ & -0.042 & 0.008 & 1.4E-07 & - & 331164 & 0 & 331164 \\
\hline $\begin{array}{l}\text { Body fat } \\
\text { percentage }\end{array}$ & -0.033 & 0.0063 & $1.5 \mathrm{E}-07$ & - & 331117 & 0 & 331117 \\
\hline $\begin{array}{l}\text { Arm fat mass } \\
\text { right }\end{array}$ & -0.041 & 0.008 & 2.5E-07 & - & 331226 & 0 & 331226 \\
\hline Trunk fat mass & -0.042 & 0.0083 & 2.9E-07 & - & 331093 & 0 & 331093 \\
\hline Pulse pressure & -0.465 & 0.0925 & 5.00E-07 & - & - & - & - \\
\hline $\begin{array}{l}\text { Arm fat } \\
\text { percentage } \\
\text { left }\end{array}$ & -0.031 & 0.0062 & 7E-07 & - & 331198 & 0 & 331198 \\
\hline $\begin{array}{l}\text { Waist } \\
\text { circumference }\end{array}$ & -0.036 & 0.0073 & 8.1E-07 & - & 336639 & 0 & 336639 \\
\hline $\begin{array}{l}\text { Self-reported } \\
\text { hypertension }\end{array}$ & -0.018 & 0.0036 & 8.2E-07 & - & 337159 & 87690 & 249469 \\
\hline
\end{tabular}




\begin{tabular}{|l|l|l|l|l|l|l|l|}
\hline $\begin{array}{l}\text { Medication for } \\
\text { cholesterol, } \\
\text { blood pressure } \\
\text { or diabetes: } \\
\text { cholesterol } \\
\text { lowering } \\
\text { medication }\end{array}$ & -0.025 & 0.0051 & $9.9 \mathrm{E}-07$ & - & 154702 & 35840 & 118862 \\
\hline $\begin{array}{l}\text { Weight } \\
\text { Arm fat }\end{array}$ & -0.035 & 0.0072 & $1.3 \mathrm{E}-06$ & - & 336227 & 0 & 336227 \\
\hline $\begin{array}{l}\text { percentage } \\
\text { right }\end{array}$ & -0.03 & 0.0062 & $1.8 \mathrm{E}-06$ & - & 331249 & 0 & 331249 \\
\hline $\begin{array}{l}\text { Trunk fat } \\
\text { percentage }\end{array}$ & -0.035 & 0.0075 & $3.9 \mathrm{E}-06$ & - & 331113 & 0 & 331113 \\
\hline
\end{tabular}

Supplementary Table 18. Pleiotropy assessment on ADH1B SNP rs1229984 using database GeneATLAS. Shortlisted traits are risk factors that are potentially associated with breast/ovarian cancer. These associations are obtained from the UK Biobank only. The GeneATLAS database is available at https://geneatlas.roslin.ed.ac.uk/phewas/)

\begin{tabular}{|l|l|l|l|l|l|}
\hline Trait & Variant & $\begin{array}{l}\text { Eff. } \\
\text { allele }\end{array}$ & beta & pvalue & MAF \\
\hline Psychological/psychiatric problem & rs1229984 & C & -0.001564 & 0.37201 & 0.0246 \\
\hline $\begin{array}{l}\text { N95 Menopausal and other } \\
\text { perimenopausal disorders }\end{array}$ & rs1229984 & C & -0.002943 & 0.12659 & 0.0246 \\
\hline Smoking status & rs1229984 & C & -0.003317 & 0.42239 & 0.0246 \\
\hline Body mass index (BMI) & rs1229984 & C & 0.17162 & $2.6542 \mathrm{e}-11$ & 0.0246 \\
\hline Standing height & rs1229984 & C & -0.026256 & 0.32622 & 0.0246 \\
\hline
\end{tabular}


Supplementary Table 19. MR association between standard drink/day alcohol consumption and breast cancer risk stratified by ER status. The MR association estimates derived from alternative MR models (other than the IVW) were shown altogether. For MR-Egger(bootstrap), 1000 bootstrap iterations were performed to obtain reliable standard errors for the causal estimates. PWM stands for the penalized weighted median model. The confidence interval of the estimated OR for ER- and ER+ breast cancer were largely overlapping and included the null $(O R=1)$, indicating minimal meaningful differences with respect to the relationship against alcohol intake.

\begin{tabular}{|c|c|c|c|c|c|c|c|c|c|}
\hline \multirow{2}{*}{ Outcome } & \multirow{2}{*}{ MR-model } & \multicolumn{4}{|c|}{$\begin{array}{l}\text { Before filtering for SNP- } \\
\text { heterogeneity }\end{array}$} & \multicolumn{4}{|c|}{$\begin{array}{l}\text { After filtering for SNP- } \\
\text { heterogeneity }\end{array}$} \\
\hline & & $\begin{array}{l}\mathrm{P} \text { - } \\
\text { value }\end{array}$ & OR & L_95Cl & U_95Cl & $\begin{array}{l}\mathrm{P} \text { - } \\
\text { value }\end{array}$ & OR & L_95Cl & U_95Cl \\
\hline \multirow[t]{4}{*}{$\begin{array}{l}\text { ER- Breast } \\
\text { cancers }\end{array}$} & MR-Egger & 0.52 & 0.93 & 0.75 & 1.15 & 0.2 & 0.89 & 0.75 & 1.06 \\
\hline & PWM & 0.22 & 0.91 & 0.78 & 1.06 & 0.22 & 0.91 & 0.78 & 1.06 \\
\hline & $\begin{array}{l}\text { IVW (random } \\
\text { effect) }\end{array}$ & 0.77 & 0.98 & 0.85 & 1.13 & 0.71 & 0.98 & 0.89 & 1.08 \\
\hline & $\begin{array}{l}\text { IVW (fixed } \\
\text { effect) }\end{array}$ & 0.71 & 0.98 & 0.88 & 1.09 & 0.74 & 0.98 & 0.88 & 1.09 \\
\hline \multirow[t]{4}{*}{$\begin{array}{l}\text { ER+ Breast } \\
\text { cancers }\end{array}$} & MR-Egger & 0.64 & 0.96 & 0.82 & 1.12 & 0.37 & 0.95 & 0.85 & 1.06 \\
\hline & PWM & 0.30 & 0.95 & 0.85 & 1.05 & 0.29 & 0.95 & 0.86 & 1.05 \\
\hline & $\begin{array}{l}\text { IVW (random } \\
\text { effect) }\end{array}$ & 0.76 & 1.02 & 0.92 & 1.12 & 0.96 & 1.00 & 0.92 & 1.08 \\
\hline & $\begin{array}{l}\text { IVW (fixed } \\
\text { effect) }\end{array}$ & 0.67 & 1.02 & 0.94 & 1.09 & 0.96 & 1.00 & 0.93 & 1.08 \\
\hline
\end{tabular}




\section{Supplementary Figures}

Supplementary Figure 1. Comparison of MR association between estimated one standard drink/day and breast cancer and EOC susceptibility using single instrument and multiple instrument approaches. Estimates for the multi-instrument analyses were derived from 34 alcohol-associated SNPs as per main analysis. LMP refer to Low-malignant potential tumours.

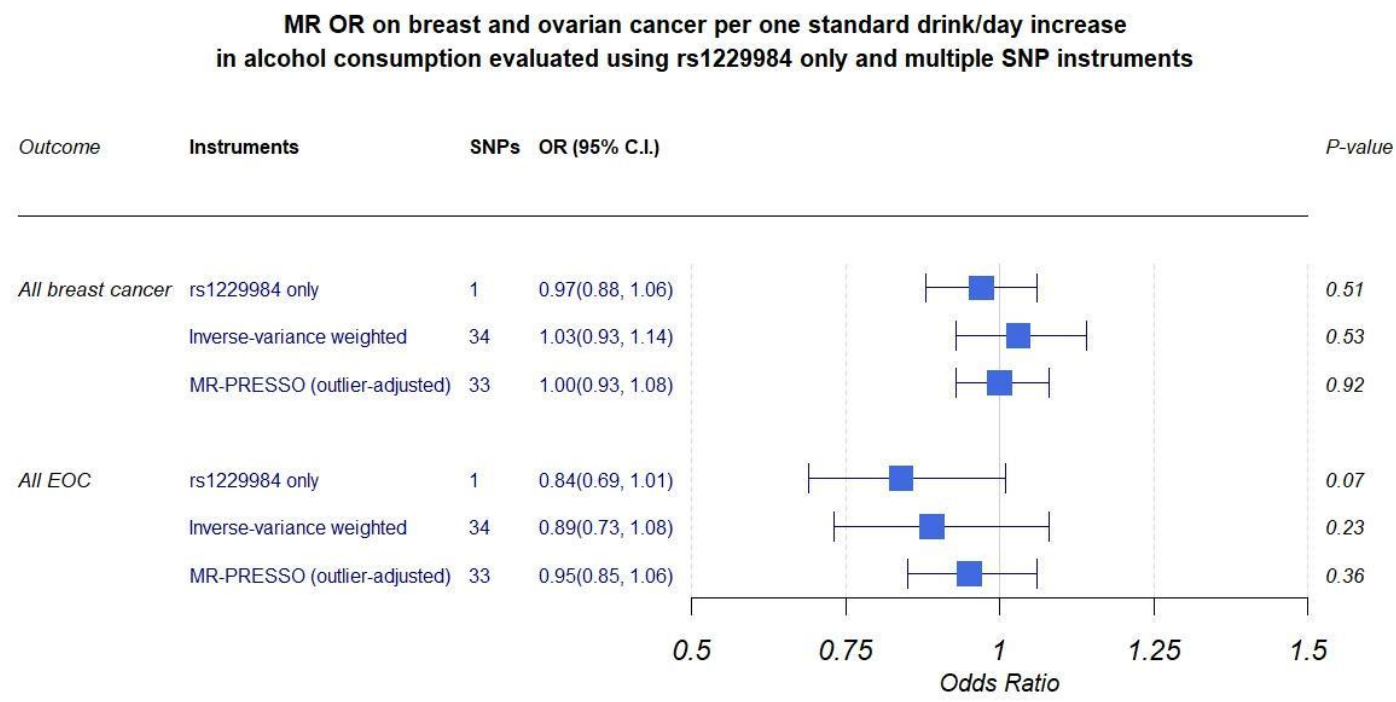

Supplementary Figure 2. MR association between estimated one standard drink/day and EOC susceptibility based on EOC subtypes. Estimates were derived from 34 alcohol-associated SNPS as per main analysis. LMP refer to Low-malignant potential tumours. The serous subtype can further be separated into high-grade serous and low-grade serous EOC.

Mendelian randomization estimates for genetically predicted one standard drink/day increase in alcohol consumption on epithelial ovarian cancer subtypes

\begin{tabular}{lrrrr} 
Outcome & Controls & Cases & P-value & OR (95\% C.I.) \\
& & & & \\
\hline & & & & \\
Serous & 40941 & 14049 & $1.45 \mathrm{e}-01$ & $0.86(0.69,1.06)$ \\
(i) High-grade serous & 40941 & 13037 & $1.61 \mathrm{e}-01$ & $0.85(0.68,1.07)$ \\
(ii) Low-grade serous & 40941 & 1012 & $7.99 \mathrm{e}-01$ & $0.94(0.58,1.52)$ \\
Mucinous & 40941 & 2566 & $2.32 \mathrm{e}-01$ & $1.20(0.89,1.61)$ \\
Clear Cell & 40941 & 1366 & $8.17 \mathrm{e}-01$ & $1.05(0.68,1.62)$ \\
Endometrioid & 40941 & 2810 & $6.10 \mathrm{e}-01$ & $0.91(0.65,1.29)$ \\
All L.M.P. EOC & 40941 & 3103 & $3.56 \mathrm{e}-01$ & $1.16(0.84,1.61)$ \\
All EOC & $\mathbf{4 0 9 4 1}$ & $\mathbf{2 2} \mathbf{4 0 6}$ & $\mathbf{2 . 2 7 e - 0 1}$ & $\mathbf{0 . 8 9}(\mathbf{0 . 7 3}, \mathbf{1 . 0 8})$
\end{tabular}

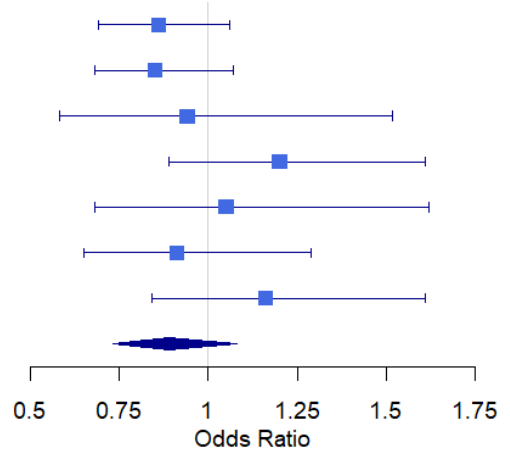


Supplementary Figure 3. Scatter plot and forest plot for the genetic association between alcohol drinks/week SNP instruments and risk of breast and ovarian cancers. The slope of the fitted line in the scatter plots reflect the MR causal estimates for each MR estimator. The forest plot shows the association of a genetically predicted one unit increase in alcohol drinks/week (need to be multiplied by 7 to obtain drink/day) on $\log (\mathrm{OR})$ of the outcome (cancer) risk inferred via each alcohol SNP instrument. The panel (a) refer to the plots for overall EOC and (b) refer to the plots for the risk of overall breast cancers respectively. The rs62055546 variant was consistently dropped after filtering for SNPheterogeneity.

(a) Overall EOC MR scatter plot and forest plot
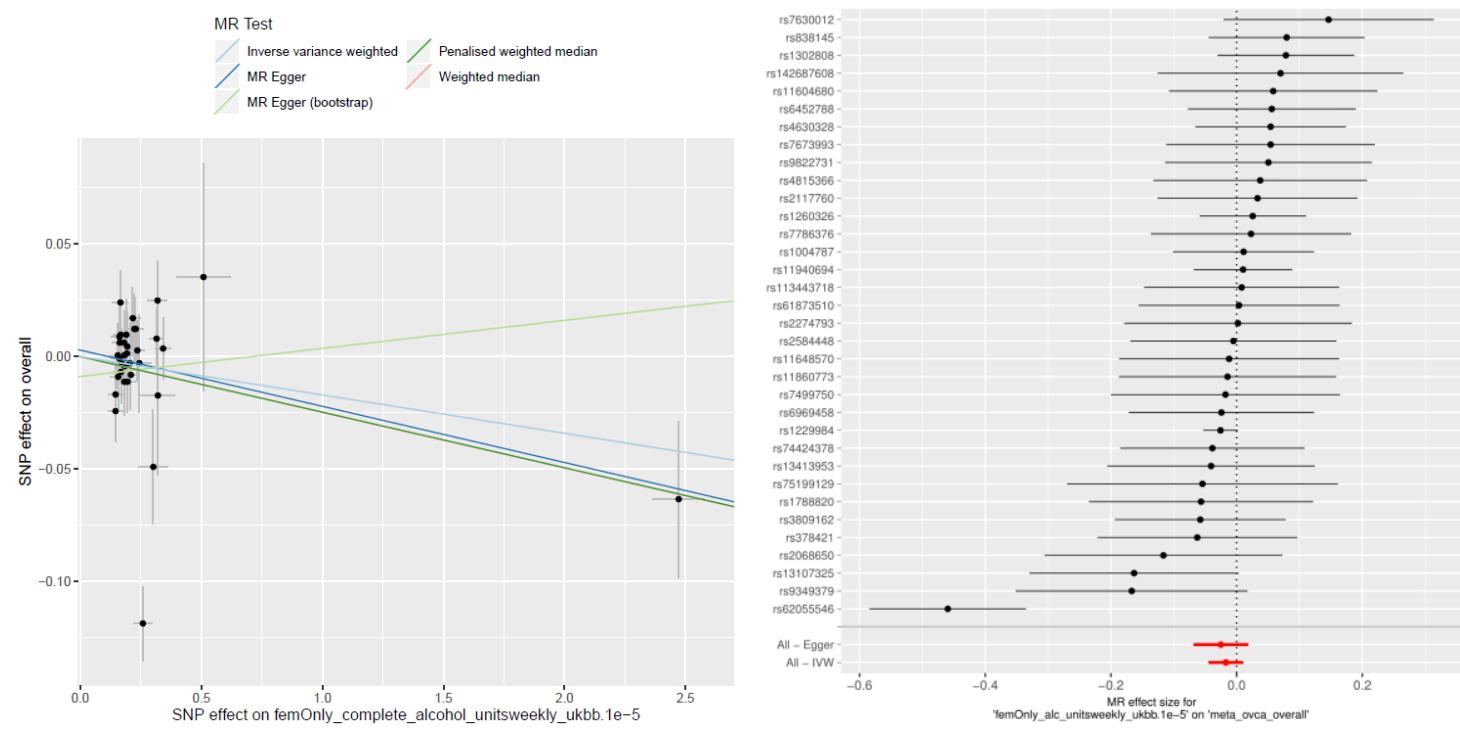

(b) All Breast cancer MR scatter plot and forest plot
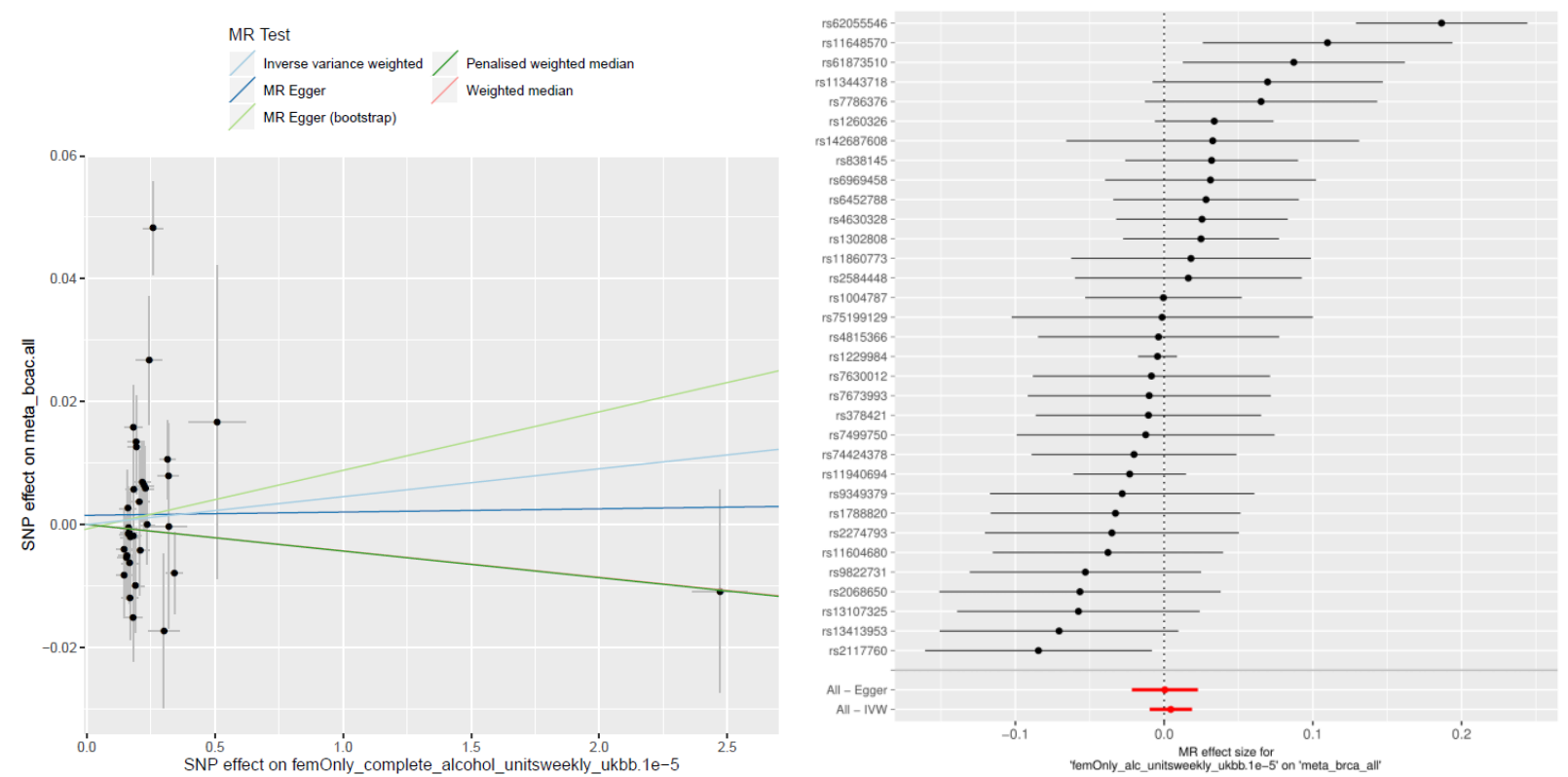
Supplementary Figure 4. MR Leave-one-out plots for the genetic association between alcohol drinks/week SNP instruments and risk of breast and ovarian cancers. The forest plot shows the IVW estimate of a genetically predicted one unit increase in alcohol drinks/week (need to be multiplied by 7 to obtain drink/day) on $\log (\mathrm{OR})$ of the outcome (cancer) risk inferred via excluding one alcohol SNP instrument at a time. The left and right side of panel (a) refer to the Leave-one-out plot for EOC and (b) refer to the plots for overall breast cancer.

\section{(a) Ovarian cancer}

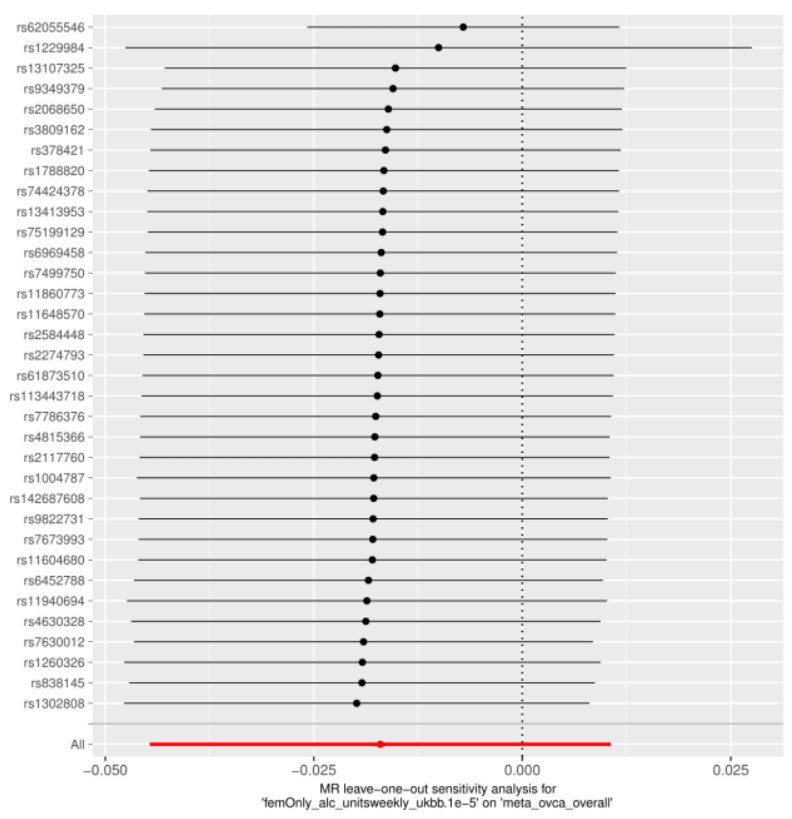

(b) MR leave-one-out breast cancer

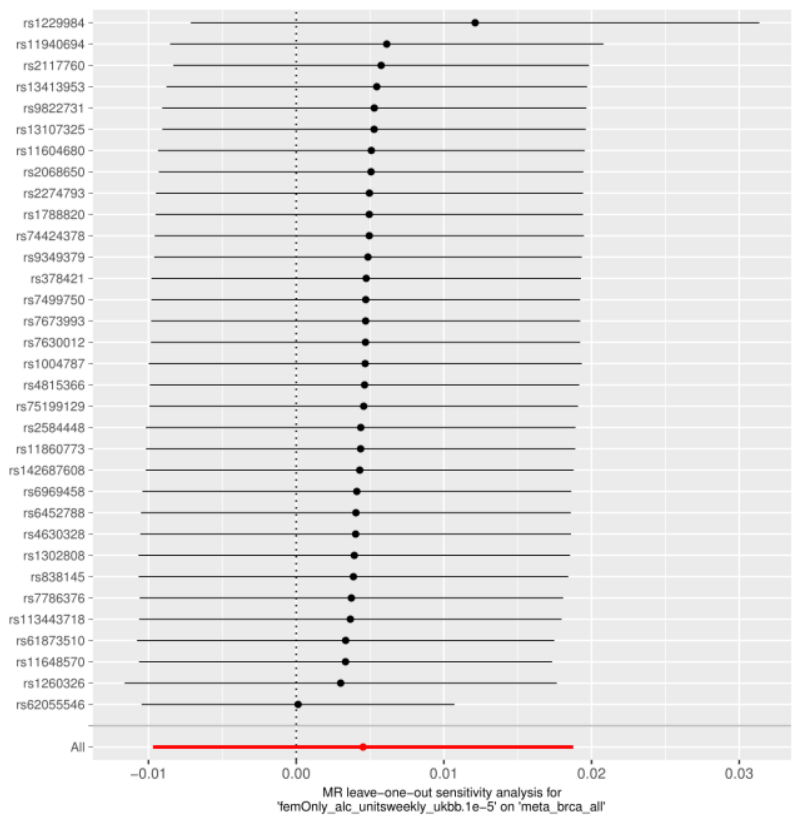


Supplementary Figure 5. Phenotypic correlation between covariates and auxilliary variables used in the multiple imputation analysis for the UKB cohort. The figure below shows the magnitude of correlation between pairs of traits, with correlations that did not achieve nominal significance $(p<0.05)$ left blank. The complete list of variables carried forward into the imputation process can be found in Table M8 (methods table in Supplementary material) .

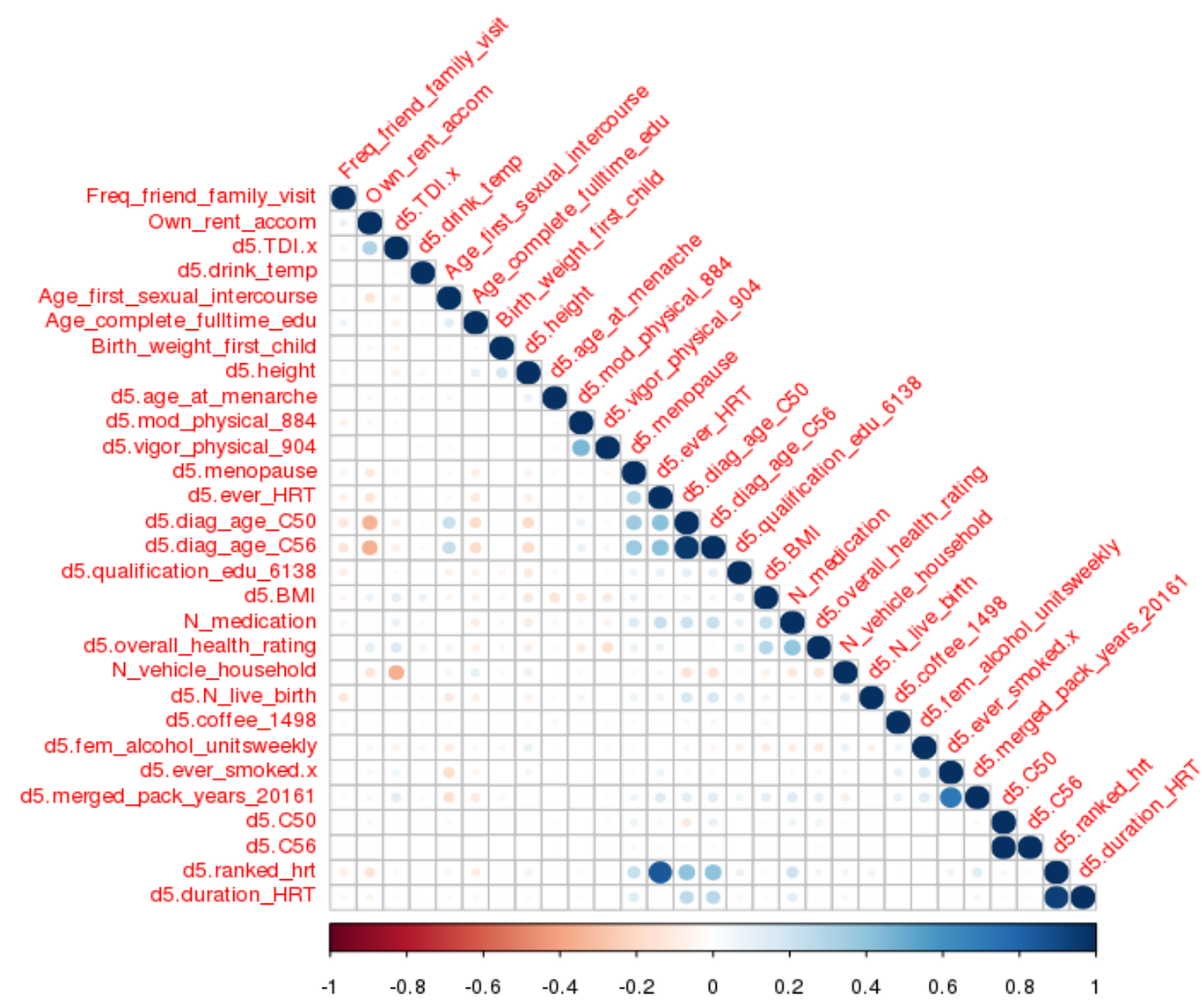




\section{Supplementary Figure 6. Indicator matrix on the predictability of covariates with missing data from}

other covariates and auxiliary variables estimated from MiCE. For each row, a positive indicator value (value $=1$ ) indicates that the trait of the corresponding column can be used to predict the row trait in a multiple imputation framework (vice versa, for column on row). The total number of predictors for a given trait is hence the sum of values across the row and column corresponding to that trait. We manually omit the cancer diagnosis outcome variables (diag_age_C50/C56 and C50/C56), genetic sex (inferred.sex) and the user ID (FID) phenotype from the multiple imputation analysis.

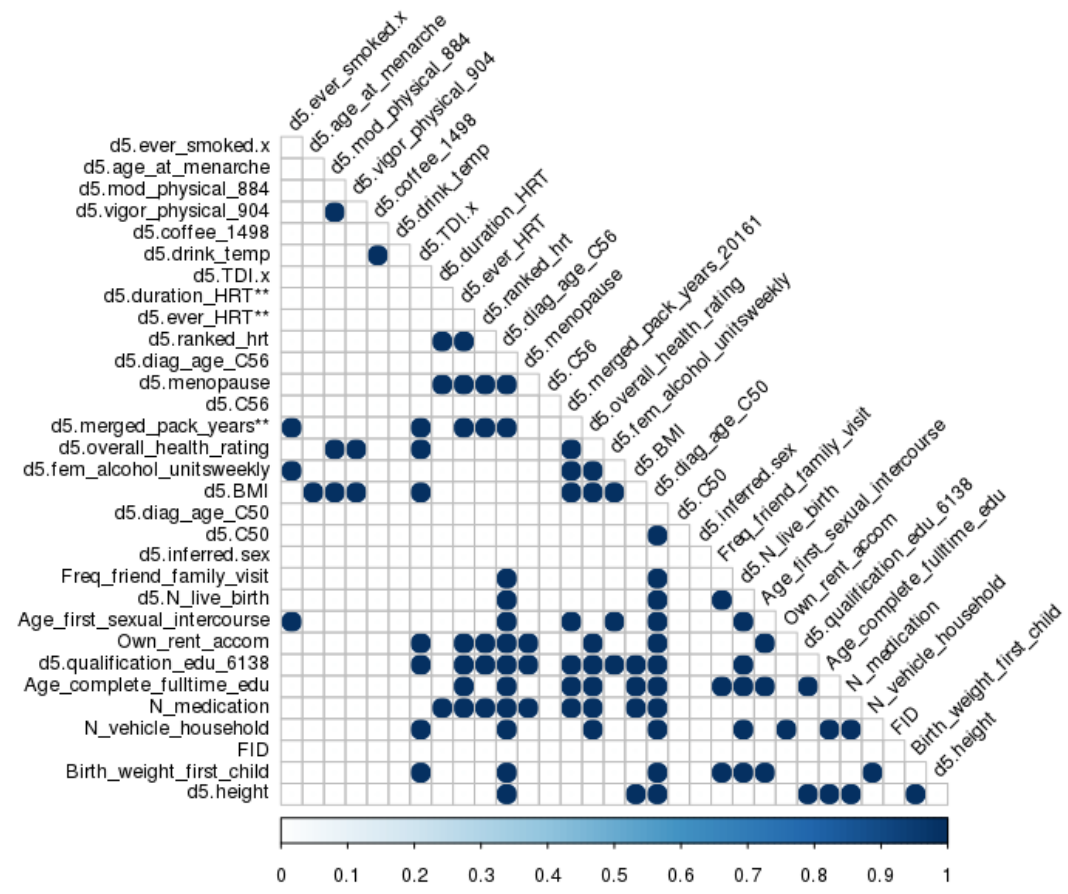




\section{Supplementary references}

1. Michailidou K, Lindström S, Dennis J, Beesley J, Hui S, Kar S, et al. Association analysis identifies 65 new breast cancer risk loci. Nature. 2017;551:92-4.

2. Clarke T-K, Adams MJ, Davies G, Howard DM, Hall LS, Padmanabhan S, et al. Genome-wide association study of alcohol consumption and genetic overlap with other health-related traits in UK Biobank (N=112 117). Mol Psychiatry. 2017;22:1376-84.

3. Burgess S, Bowden J, Fall T, Ingelsson E, Thompson SG. Sensitivity Analyses for Robust Causal Inference from Mendelian Randomization Analyses with Multiple Genetic Variants. Epidemiology. 2017;28:30-42.

4. Verbanck $M$, Chen $C-Y$, Neale B, Do R. Detection of widespread horizontal pleiotropy in causal relationships inferred from Mendelian randomization between complex traits and diseases. Nat Genet. 2018;50:693-8.

5. Hemani G, Zheng J, Elsworth B, Wade KH, Haberland V, Baird D, et al. The MR-Base platform supports systematic causal inference across the human phenome. Elife [Internet]. 2018;7. Available from: http://dx.doi.org/10.7554/eLife.34408

6. Yavorska OO, Burgess S. MendelianRandomization: an R package for performing Mendelian randomization analyses using summarized data. Int J Epidemiol. 2017;46:1734-9. 
White British Individuals with self-reported alcohol data in the UK Biobank

Estimated standard drinks/week GWAS for

(i) both sex, (ii) females only

Identified 78 SNP instruments associated with

est. std drinks/week in combined sex alcohol

GWAS

Used 34 out of 78 SNPs that were replicated in women only alcohol GWAS $(p<1 e-5)$ for main MR analyses

\section{Perform two-sample Mendelian randomization}

SNP-alc effect sizes estimated from females only

Single instrument MR using rs1229984
Extract SNP-cancer association estimates from cancer GWASs

$(B C A C+O C A C)$

Multi-instrument MR using 34 SNPs 
Meta-analysis of observational hazard ratio estimates for one standard drink increase on risk of breast and ovarian cancers

$\begin{array}{llll}\text { Studies } & \text { Cancers } & \text { Cases; Total } & \text { HR }(95 \% \text { C.I.) }\end{array}$

$\begin{array}{lrrr}\text { CCHS+CGPS } & \text { Breast } & 2039 ; 65126 & 1.09(1.05,1.13) \\ \text { KARMA } & \text { Breast } & 985 ; 60903 & 1.07(0.97,1.19) \\ \text { UKBB } & \text { Breast } & 1787 ; 62867 & 1.04(1.01,1.07) \\ \text { Combined (BrCa) } & \text { Breast } & \mathbf{4 8 1 1 ; 1 8 8 8 9 6} & \mathbf{1 . 0 6}(\mathbf{1 . 0 4 , 1 . 0 8 )} \\ \text { CCHS+CGPS } & \text { EOC } & 264 ; 61427 & 1.07(0.96,1.20) \\ \text { UKBB } & \text { EOC } & 187 ; 61267 & 0.92(0.83,1.03) \\ \text { Combined (EOC) } & \text { EOC } & \mathbf{4 5 1 ; 1 2 2 9 6 4} & \mathbf{1 . 0 0}(\mathbf{0 . 9 2}, \mathbf{1 . 0 8})\end{array}$

$\begin{array}{lcccc}0.5 & 0.75 & 1 & 1.25 & 1.5\end{array}$


Mendelian randomization estimate for the odds ratio on breast and ovarian cancer per one standard drink/day increase in estimated alcohol consumption

Outcome

Instruments

SNPS OR (95\% C.I.)

P-value

All breast cancer Inverse-variance weighted

34

$1.03(0.93,1.14)$

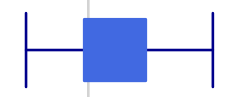

0.53

MR-PRESSO (outlier-adjusted) 33

$1.00(0.93,1.08)$

$+1$

MR-Egger

$34 \quad 1.00(0.86,1.17)$

Penalised weighted median

34

$0.97(0.89,1.06)$

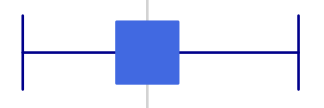

0.92

0.96

0.5

EOC

$\begin{array}{lrl}\text { Inverse-variance weighted } & 34 & 0.89(0.73,1.08) \\ \text { MR_PRESSO (outlier-adjusted) } 33 & 0.95(0.85,1.06) \\ \text { MR-Egger } & 34 & 0.84(0.62,1.14) \\ \text { Penalised weighted median } & 34 & 0.84(0.70,1.01)\end{array}$

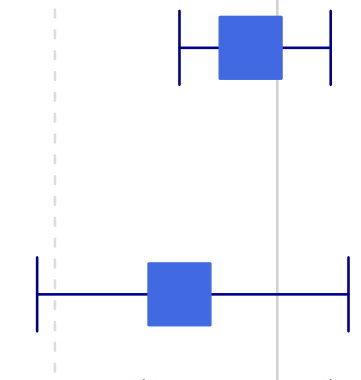

0.23

0.36

0.27

\section{:}

0.5

0.5

0.75

Odds Ratio

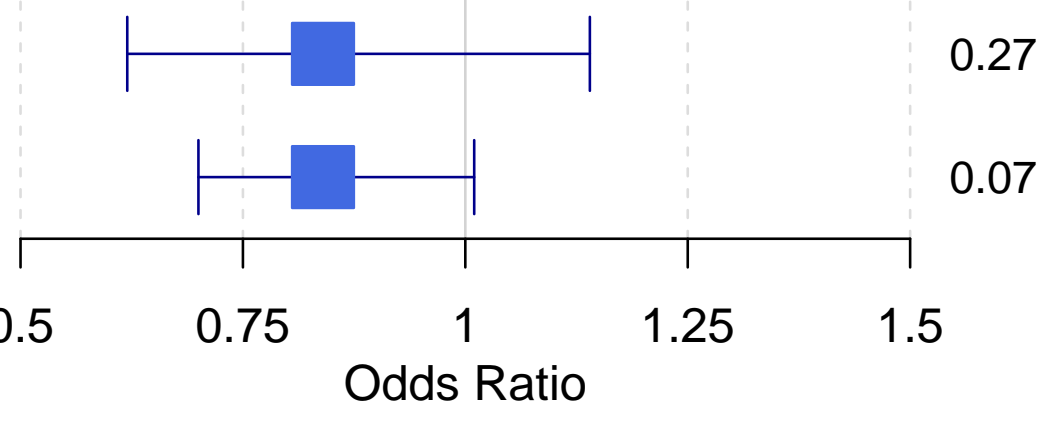


Comparison of observational and genetic estimates for one standard drink/day increase in alcohol consumption on risk of breast and ovarian cancers

$\begin{array}{llll}\text { Studies Cancers Cases Model Measure } & \text { Estimate } \\ (95 \% \text { C.I.) }\end{array}$

\begin{tabular}{|c|c|c|c|c|c|}
\hline Observational & Breast & 4811 & $\mathrm{HR}$ & 1 std drink/day & $1.06(1.04,1.08)$ \\
\hline WCRF CUP meta-analysis & Breast & 35221 & $\mathrm{RR}$ & $10 \mathrm{~g}$ ethanol/day & $1.09(1.07,1.12)$ \\
\hline MR analysis (BCAC data) & Breast & 122977 & OR & 1 std drink/day & $1.00(0.93,1.08)$ \\
\hline Observational (HR) & EOC & 451 & $\mathrm{HR}$ & 1 std drink/day & $1.00(0.92,1.08)$ \\
\hline WCRF SLR meta-analysis & EOC & 2954 & $\mathrm{RR}$ & $10 \mathrm{~g}$ ethanol/day & $1.01(0.96,1.06)$ \\
\hline MR analysis (OCAC data) & EOC & 22406 & OR & 1 std drink/day & $0.95(0.85,1.06)$ \\
\hline
\end{tabular}

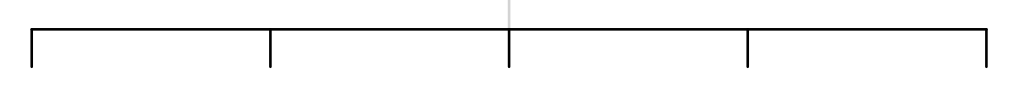

$0.5 \quad 0.75 \begin{array}{lll}1 & 1.25 & 1.5\end{array}$




\section{MR Test}

Inverse variance weighted (fixed effects)

Inverse variance weighted (multiplicative random effects)

MR Egger (bootstrap)

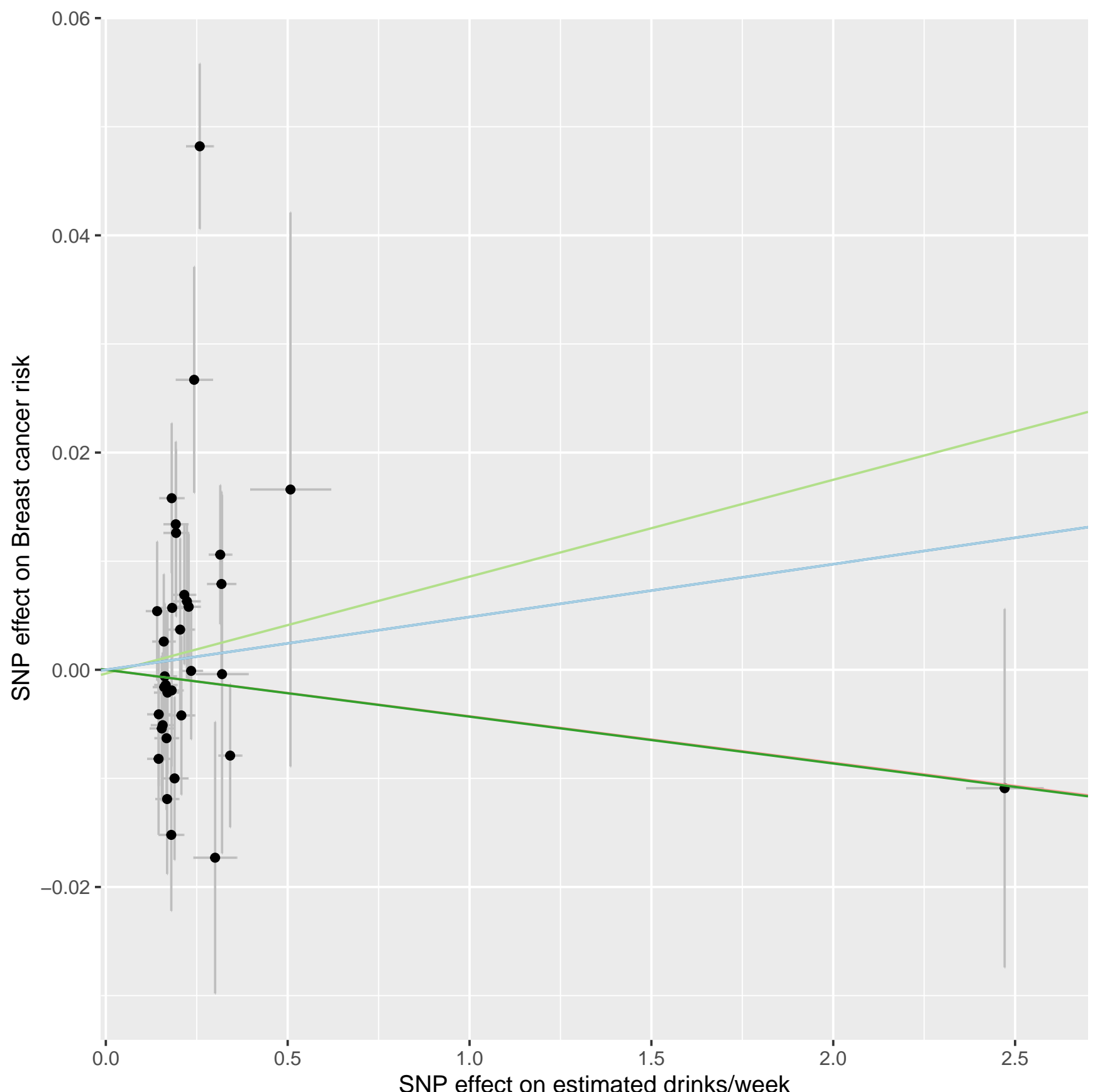

Penalised weighted median

Weighted median
B

MR Test

Inverse variance weighted (fixed effects)

Penalised weighted median

Inverse variance weighted (multiplicative random effects)

MR Egger (bootstrap)

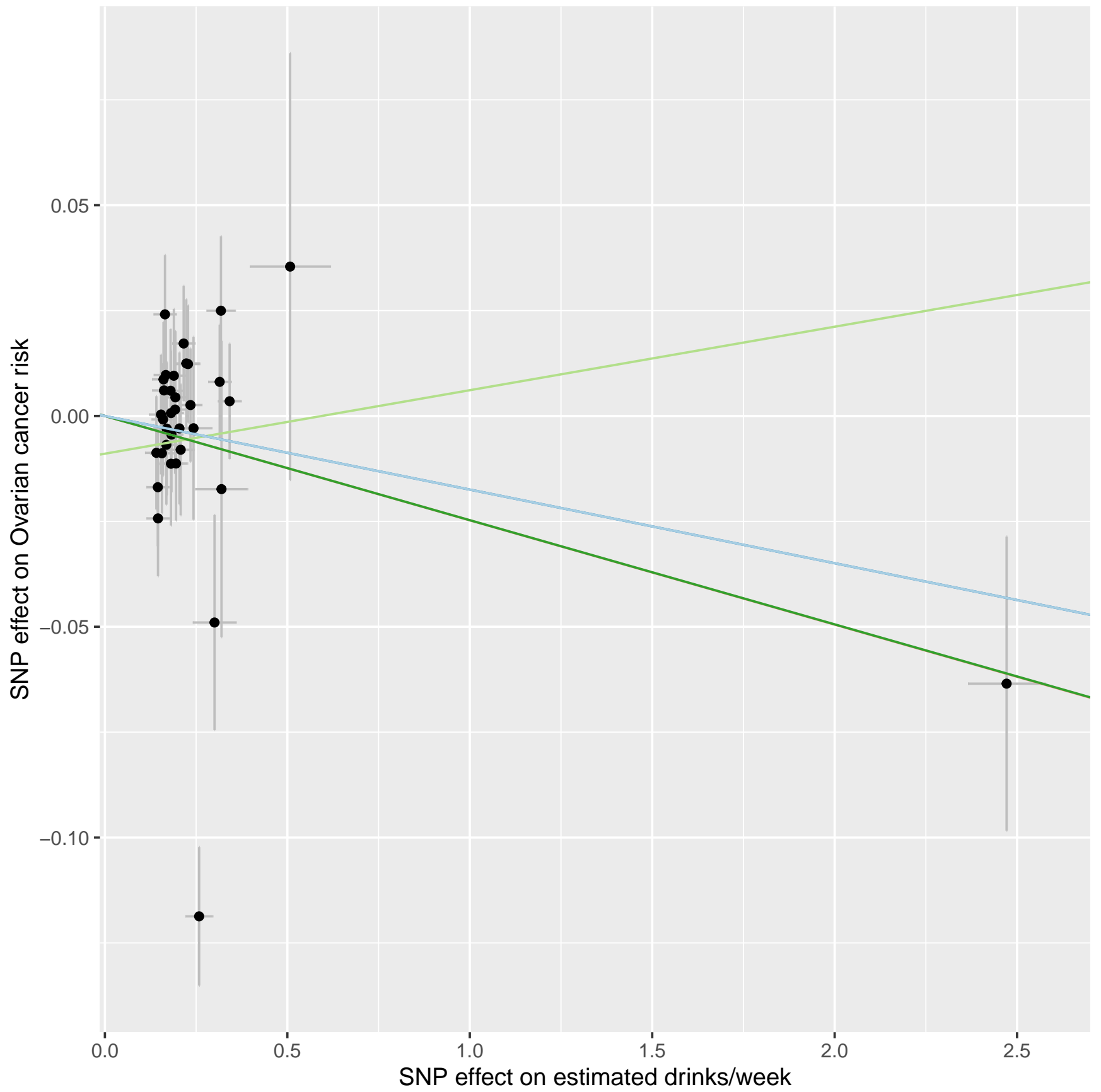

